OPEN ACCESS

Edited by: Urs Granacher,

University of Potsdam, Germany

Reviewed by: Beat Knechtle,

University Hospital Zurich, Switzerland

Frantisek Zahalka,

Charles University, Czechia

*Correspondence:

Erika Zemková

erika.zemkova@uniba.sk

tORCID:

Erika Zemková

orcid.org/0000-0003-0938-5691

Zuzana Kováčiková

orcid.org/0000-0002-9206-7725

Specialty section:

This article was submitted to

Exercise Physiology,

a section of the journal

Frontiers in Physiology

Received: 23 February 2020

Accepted: 02 July 2020

Published: 24 July 2020

Citation:

Zemková E, Kováčiková Z and

Zapletalová L (2020) Is There

a Relationship Between Workload

and Occurrence of Back Pain

and Back Injuries in Athletes?

Front. Physiol. 11:894.

doi: 10.3389/fphys.2020.00894

\section{Is There a Relationship Between Workload and Occurrence of Back Pain and Back Injuries in Athletes?}

\author{
Erika Zemková ${ }^{1,2,3 * \dagger}$, Zuzana Kováčiková ${ }^{4 \dagger}$ and Ludmila Zapletalová ${ }^{3}$ \\ ${ }^{1}$ Department of Biological and Medical Sciences, Faculty of Physical Education and Sports, Comenius University \\ in Bratislava, Bratislava, Slovakia, ${ }^{2}$ Sports Technology Institute, Faculty of Electrical Engineering and Information Technology, \\ Slovak University of Technology, Bratislava, Slovakia, ${ }^{3}$ Institute of Physiotherapy, Balneology and Medical Rehabilitation, \\ University of Ss. Cyril and Methodius in Trnava, Trnava, Slovakia, ${ }^{4}$ Department of Natural Sciences in Kinanthropology, \\ Faculty of Physical Culture, Palacký University Olomouc, Olomouc, Czechia
}

The back is subjected to a great deal of strain in many sports. Up to $20 \%$ of all sports injuries involve an injury to the lower back or neck. Repetitive or high impact loads (e.g., running, gymnastics, skiing) and weight loading (e.g., weightlifting) affect the lower back. Rotation of the torso (e.g., golf, tennis) causes damage to both, the lumbar and thoracic spine. The cervical spine is most commonly injured in contact sports (e.g., boxing, football). One of the factors that increases the odds of injuries in athletes is excessive and rapid increases in training loads. In spite of currently emerging evidence on this issue, little is known about the balance between physiological loading on the spine and athletic performance, versus overloading and back pain and/or injury in athletes. This scoping review aims (i) to map the literature that addresses the association between the training load and the occurrence of back pain and/or injury, especially between the Acute:Chronic Workload Ratio (ACWR) and back problems in athletes of individual and team sports, and (ii) to identify gaps in existing literature and propose future research on this topic. A literature search of six electronic databases (i.e., MEDLINE, PubMed, Web of Science, SCOPUS, SportDiscus, and CINAHL) was conducted. A total of 48 research articles met the inclusion criteria. Findings identified that fatigue of the trunk muscles induced by excessive loading of the spine is one of the sources of back problems in athletes. In particular, high training volume and repetitive motions are responsible for the high prevalence rates. The most influential are biomechanical and physiological variations underlying the spine, though stress-related psychological factors should also be considered. However, limited evidence exists on the relationship between the ACWR and back pain or non-contact back injuries in athletes from individual and team sports. This may be due to insufficiently specified the acute and chronic time window that varies according to sport-specific schedule of competition and training. More research is therefore warranted to elucidate whether ACWR, among other factors, is able to identify workloads that could increase the risk of back problems in athletes.

Keywords: Acute:Chronic Workload Ratio, back problems, individual sports, team sports, training load 


\section{INTRODUCTION}

Back injuries and back pain are among the most common health problems in athletes affecting their performance (Trompeter et al., 2017). Acute injury or pain can be caused by falling, being tackled in team sports, fighting in combat sports, or while lifting heavy weights (Caine and Nassar, 2005; Aasa et al., 2017; Bromley et al., 2018; Plais et al., 2019). However, much more common than acute incidents are chronic back problems (Alzahrani et al., 2019). In many sports, the back and spinal column undergoes elevated stress for a long time (Lawrence et al., 2006). This may result in inflammation around the vertebrae and back muscles, which sometimes causes injuries to the discs but much more often leads to upper or lower back pain (Trompeter et al., 2017). Sciatica, for instance, is back pain that also affects the back of the legs or even the feet (Koes et al., 2007). It can occur in cyclists who are in a flexed forward posture or athletes of water and swing sports who perform a great deal of trunk rotation. Particularly in sports with repetitive asymmetric loading, the sacroiliac joint dysfunction is a frequent cause of low back pain in athletes (Peebles and Jonas, 2017). In most of these sports, asymmetric loading causes side-to-side dysbalances that may greatly enhance susceptibility to back pain. A clear example is an association between repeated golf swings and golf-related low back injuries (Cole and Grimshaw, 2016). Trunk rotational power represents the measure that reflects this asymmetric loading during trunk rotation (Zemková et al., 2019a). Its values were found to be significantly higher on the dominant than nondominant side in golfers $(\sim 15 \%)$, tennis $(\sim 12 \%)$ and hockey players $(\sim 14 \%)$ at lower or higher loads, whereas there were no significant between-side differences in a control group of physically active subjects $(\sim 7 \%)$. This parameter is also able to reflect the specificity of training programs in the preparatory and competitive periods in canoeists, hockey and tennis players (Poór and Zemková, 2018). Similarly, wheelchair athletes, who suffer from spine curvature disorders, are at greater risk of back problems (Samuelsson et al., 2001). This is related to mainly those participating in sports that involve trunk rotational motions under loading and unloading conditions (Goosey-Tolfrey, 2010). A recent study demonstrated that wheelchair table tennis players exhibit lower mobility in the thoracic and lumbar regions of the spine during trunk flexion as well as lower lumbar inversion and pelvic retroversion than able-bodied athletes (Zemková et al., 2018b). Their limited range of motion (ROM) during trunk rotations and decreased posterior concavity contributes to lower trunk rotational velocity (Zemková et al., 2018b) and it may also increase the risk of spine injury. Velocity of trunk rotational movement is also compromised in older athletes, most likely due to their reduced ROM as a result of increased trunk stiffness with aging (Zemková et al., 2018a).

In spite of the fact that low back pain in athletes is among the most prevalent musculoskeletal condition with incidence rates of $1-30 \%$, it is often neglected in research studies (Graw and Wiesel, 2008). The prevalence of low back pain is 1-94\%, (the highest in rowing and cross-country skiing), and its point prevalence is $18-65 \%$ (the highest in rowing and the lowest in basketball) (Trompeter et al., 2017). Prevalence rates vary during an athlete's season with the highest rates observed during the peak season (Newlands et al., 2015). The evidence on risk factors is mostly restricted to lumbar spine and hip flexibility, back muscle strength, trunk extensor/flexor endurance, and some anthropometric characteristics (Moradi et al., 2015; Trompeter et al., 2017). However, less attention has been paid to sport related factors. Among those, high training volume and repetitive motions contribute to the high prevalence rates (Fett et al., 2017). Athletes involved in impact sports are at risk for certain spinal pathologies that are associated with repetitive loading of the spine (Lawrence et al., 2006). For instance, higher incidence rates of spondylolysis and degenerative disk disease have been reported in elite athletes who participate in more intense training over a longer period of time than those who do not (Lawrence et al., 2006). Though muscle fatigue is often a symptom of back pain in athletes, factors like the number of training sessions per week only rarely have been investigated (Fett et al., 2017). The concept that compares the amount of acute workload performed in a 1week relative to a 4-week chronic workload can provide useful information for designing the optimal workload that would improve athlete performance, and at the same time, decrease the likelihood of back pain and related injuries.

The Acute:Chronic Workload Ratio (ACWR) is a simplified version of the fitness-fatigue model that was first introduced by Banister et al. (1975). It is definied as the ratio between training loads in recent periods ( $\sim 5-10$ days) and over longer periods ( 4-6 weeks) (Foster et al., 1999; Hulin et al., 2016b). The ACWR is calculated by using the rolling or exponentially weighted moving average model (Hunter, 1986). Though both models are used in practice, the first one is unable to deal with the decaying nature of fatigue and fitness effects over time and the non-linearity of workload and the occurrence of injury; thus it may less precisely reflect variations of accumulated loads (Williams et al., 2017; Menaspa, 2017). The second one, which attributes a decreasing weighting in order to compensate for the load latency effects (Williams et al., 2017), is more sensitive to detect increases in injury risk at higher ACWR ranges during the preseason and in-season periods (Griffin et al., 2020).

These models have been used to better control and understand the training process. Several studies have documented the association between the actual and modeled performance in a variety of individual sports (e.g., Taha and Thomas, 2003; Jobson et al., 2009) or injuries in team sports (e.g., Hulin et al., 2016a; Bowen et al., 2017; Murray et al., 2017). Contrary to this, less attention has been paid to the investigation and use of these models in association with the occurrence of injuries or pain that especially involve the spine and back muscles. So far the training volume, internal and external loads in different types of training, sport-specific demands on the spine in relation to back pain and/or injury have been primarily investigated (e.g., Maselli et al., 2015; Newlands et al., 2015; van Hilst et al., 2015). There is however, no sufficient evidence to determine the relationship between ACWR and the occurrence of back problems in athletes. A question also remains as to whether ACWR can be predictive of an athlete's risk of back pain and/or injury.

To this end, two main questions were addressed in this scoping review: (1) Is the training load associated with the occurrence of 
back problems in athletes of individual and team sports? (2) Is there a relationship between the ACWR and back pain and/or injury in these athletes? Accordingly, this scoping review aimed at (i) mapping the literature that addresses the association between the training load and the occurrence of back pain and/or injury, especially between the ACWR and back problems in athletes of individual and team sports, and (ii) identifying gaps in existing literature and proposing future research on this topic.

\section{METHODS}

The article was designed as a scoping review (Armstrong et al., 2011). In order to answer the above questions and to identify a gap in existing research in the field, a literature search was provided. Electronic literature was searched on MEDLINE, PubMed, Web of Science, SCOPUS, SportDiscus, and CINAHL databases. Further searches were conducted on Elsevier, SpringerLink, EBSCOhost, and Google Scholar. Besides articles in peer-reviewed journals, also conference proceedings were analyzed. The search was confined to studies closely associated with the major topic of this review, i.e., investigating the relationship between training loads and the occurrence of back problems in athletes, particularly between the ACWR and back pain and/or injury in athletes of individual and team sports. Our primary focus was concentrated on outcome measures of the ACWR and data related to back problems in athletes of individual and team sports. Using this approach, however, led to the identification of a limited number of studies that were able to meet the eligibility criteria for this review. Therefore the search was later widened to include all relevant studies that investigated the relationship between the training load and back pain and/or injury in athletes. Together these help us to identify gaps in the current literature regarding the training load, especially the ACWR and their associations with back pain and/or injury in athletes of individual and team sports, and suggest a proposal for future research.

The target population was athletes of individual (cricket, cycling, dancing, diving, golf, gymnastics, horseback riding, jiu jitsu, judo, powerlifting, rowing, running, skiing, swimming, triathlon, weightlifting, wrestling) and team sports (basketball, florball, handball, hockey, soccer, tennis, volleyball). The most frequent terms “Acute:Chronic Workload Ratio," "back injuries," "back pain," "back problems," "competition," "external training load," "fitness level," "injury risk," "internal training load," "low back pain," "neck/cervical pain," "non-contact injury," "thoracic pain," and "training" were combined with particular individual and team sports. Additional searches were performed by using the words from subheadings, such as factors contributing to back pain and/or injury in athletes with respect to their localization, intensity, frequency, and duration. The key inclusion criterion was that studies involved athletes of individual and team sports with back pain and/or injury, a specified training program, and objective or subjective measures relevant to this review. Studies were excluded if they were incomplete (abstracts, etc.), not peerreviewed, did not contain original research, and were not written in the English. Studies that failed to meet these criteria for this review were excluded. Figure 1 represents particular phases of the search process.

\section{RESULTS AND DISCUSSION}

\section{Overview of Studies Dealing With Back Pain and/or Injury in Athletes of Individual Sports}

The key data elements that were sought for each study were categorized as follows: (1) study design, (2) study population (number of athletes, sex, age, type of sport, and performance
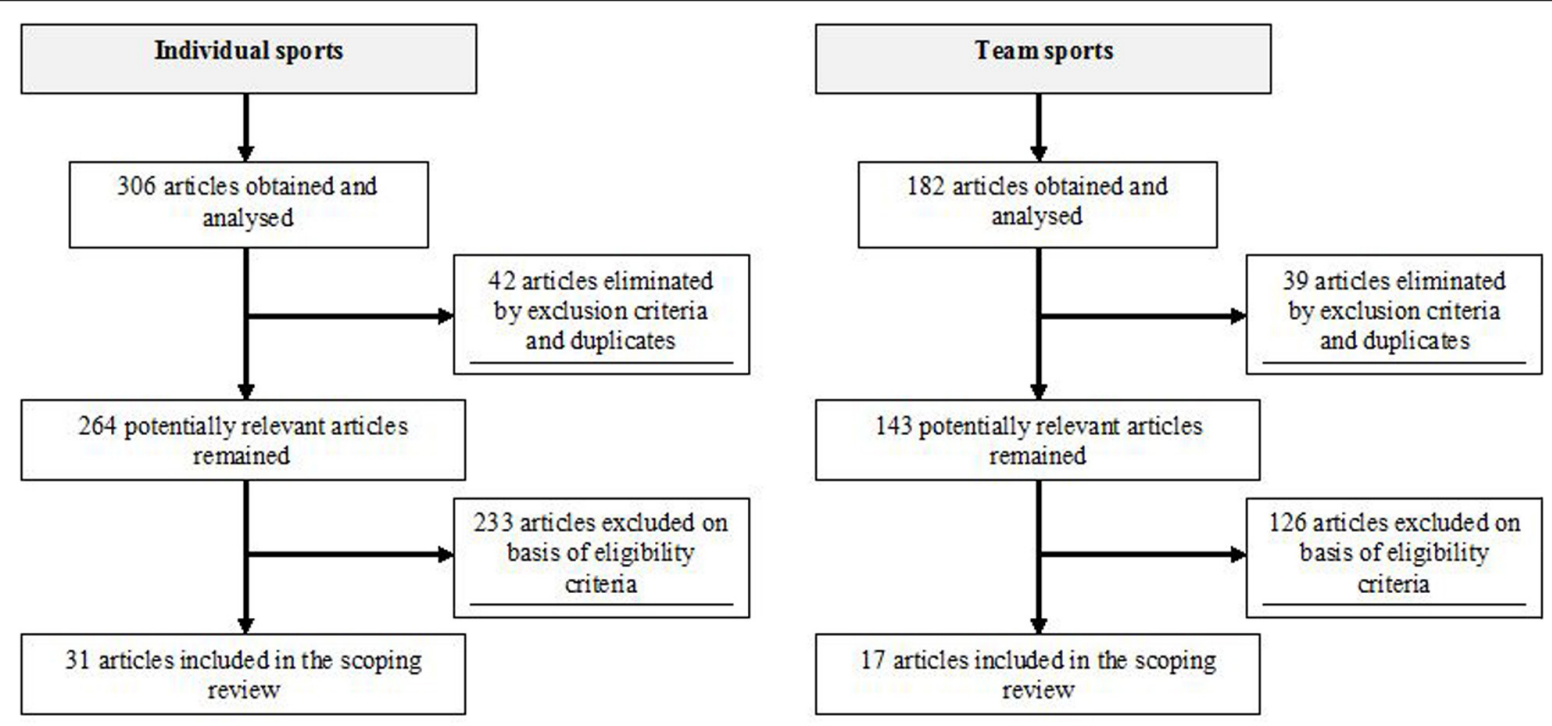

FIGURE 1 | Flow chart illustrating phases of the literature search and study selection. 
level), (3) training characteristics, (4) low back pain information, (5) diagnostic methods used for identification of lumbar spine, low back pain and lower back injuries, and (6) study outcomes.

Out of the 31 research articles (Table 1), 21 studies (68\%) included both sexes, six studies (19\%) dealt with male athletes, and four studies (13\%) focused on female athletes. A total of 2999 athletes of mean age of 26.1 years was evaluated, out of which 1819 were males and 1180 were females. Fifteen studies were conducted with elite athletes, nine studies assessed non-elite athletes, and six studies were conducted in both elite and non-elite athletes. For the purpose of this review, elite athletes were defined as those competing professionally at national level, international level or at the Olympic Games. Athletes competing at district and regional competition levels, as well as recreational and amateur athletes were defined as non-elite. Also athletes competing at high schools, colleges, or universities were considered as non-elite.

Regarding the individual sports, these groups of athletes were included: cricket bowlers (1 study), cyclists (5), dancers (2), divers (1), golfers (4), gymnasts (4), horseback riders (1), jiu jitsu athletes (1), judoists (1), powerlifters (1), rowers (2), runners (2), skiers (3), swimmers (2), triathletes (1), weightlifters (1), and wrestlers (1). Studies included athletes from the following countries: Australia (3 studies), Belgium (1), Brazil (1), Colorado (1), England (1), Germany (2), Greece (1), Italy (1), Japan (3), Korea (1), Poland (1), Spain (1), Sweden (5), Switzerland (1), and Zimbabwe (1). Country of origin was not specified in seven studies.

Lumbar spine abnormalities and alterations, low back injuries and pain were diagnosed in many different ways: using imaging systems such as magnetic resonance imaging (MRI), radiographs, CT, Spinal Mouse, motion analysis, ultrasonic measurements, through clinical examination, physical examination, various questionnaires, surveys, body charts, interviews, pain scales, and combinations of the above.

\section{Overview of Studies Dealing With Back Pain and/or Injury in Athletes of Team Sports}

Out of the 17 research articles (Table 2), seven studies (41\%) included both sexes, six (35\%) were focused on male and four $(24 \%)$ on female athletes. The number of athletes in respective studies ranged from 29 to 1110 depending on the research methods used (retrospective vs. prospective). Ten out of these studies (56\%) dealt with senior players, seven (39\%) with junior players and one study (5\%) with both age categories. Participants were top athletes (4 studies), elite players (4), nonelite players of different performance levels (5), and players of more than one performance level (5). Top athletes were considered those competing at the international level and taking part in top international competitions (Olympic Games, World Tours, and World Championships). Elite athletes were defined as the members of national teams not competing at the highest level or young athletes who were members of academies for talented youth. Non-elite athletes were from sport clubs, high schools, and colleges.

Regarding the team sports category, the following groups of athletes were included: soccer players (7 studies), volleyball players (5), beach volleyball and handball players (3), field hockey, floorball, handball and basketball players (2), and the players of different types of rugby (1). Studies included were from the following countries: Norway (4 studies), Germany (3), United Kingdom, Sweden, and Finland (2), Switzerland, United States, Australia, Iran, and Portugal (1). Players included were from the same countries except of three studies which were conducted at major international events such as World Championships or World Tours.

This review includes two main types of studies: prospective cohort studies and retrospective studies. Low back injuries and pain or lumbar spine abnormalities were diagnosed in the following ways: using imaging systems (MRI), through physical examination by medical staff or physiotherapists, questionnaires, interviews, and their various combinations.

\section{The Association of Workload With the Occurrence of Back Problems in Athletes of Individual and Team Sports}

There is no conclusive evidence to date to demonstrate that the cause of low back pain in athletes of individual sports lies in undue abnormalities of the spine. Only one study (Koyama et al., 2013) confirmed lumbar disc degeneration as a statistically significant variable accounting for low back pain. However, no direct association between the training load and back injuries or abnormalities in the spine was confirmed. Nonetheless, there were trends toward an increased risk of injuries with increasing levels of performance and high physical loads.

Based on the MRI studies (Tertti et al., 1990; Baranto et al., 2006; Kaneoka et al., 2007; Kraft et al., 2009; Koyama et al., 2013; Sekine et al., 2014; Thoreson et al., 2017b; Witwit et al., 2018), the most common abnormalities in athletes of individual sports were as follows: reduced disc signal, degenerated discs at various disc levels, ring apophyses injury, disc bulging, Schmorl's nodes causing severe back pain, abnormal configuration of the vertebra, and alterations in spinal curvatures.

In team sport games, the prevalence of overuse low back injuries accompanied by low back pain is rather high. However, the research findings are inconsistent. The level of prevalence depends on the type of research design used. While in prospective studies the prevalence of low back pain was evident in 9-20\% of the cases (Bahr and Reeser, 2003; Jacobson and Tegner, 2007; Pasanen et al., 2008; Bere et al., 2015; Aasheim et al., 2018) or 2.25-0.4/1000 training and match hours (Bacon and Mauger, 2017; Bowen et al., 2017), in the retrospective studies it occurred in $15-97 \%$ of the cases depending on time to occurrence of back problems (point, 1-week, 1-, 3-, 12-month, life time prevalence) (Hoskins et al., 2009; Haydt et al., 2012; Külling et al., 2014; Leppänen et al., 2015; Tunås et al., 2015; Haag et al., 2016; Farahbakhsh et al., 2018; Fett et al., 2019).

The highest prevalence of low back pain was identified in field hockey, floorball, rugby, and beach volleyball (Nosco et al., 
TABLE 1 | Training characteristics and identified back problems in athletes of individual sports.

\begin{tabular}{|c|c|c|c|c|c|c|}
\hline Authors & $\begin{array}{l}\text { Sport and } \\
\text { performance } \\
\text { level }\end{array}$ & Subjects & Training & Back pain and/or injury & $\begin{array}{l}\text { Diagnostics of } \\
\text { back pain and/or } \\
\text { injury }\end{array}$ & Main findings \\
\hline Baranto et al., 2006 & $\begin{array}{l}\text { - } 20 \text { Swedish elite } \\
\text { divers ( } 14 \text { girls and } \\
6 \text { boys), still active } \\
\text { in competition } \\
\text { - } 18 \text { divers active in } \\
\text { competitive diving } \\
\text { at follow-up }\end{array}$ & $\begin{array}{l}\text { Age at baseline: } \\
16.4 \pm 3.1 \text { years } \\
\text { Age at follow-up: } \\
21.2 \pm 3.0 \text { years }\end{array}$ & $\begin{array}{l}\text { Training days/week at } \\
\text { follow-up: } 4.0 \pm 1.6 \mathrm{~h} \\
\text { Training hours/week at } \\
\text { follow-up: } 6.5 \pm 2.9 \mathrm{~h}\end{array}$ & $\begin{array}{l}\text { Back pain was self-assessed. It } \\
\text { was graded as moderate if it } \\
\text { does not affect training and } \\
\text { competition. If it affects training } \\
\text { and competition it was graded } \\
\text { as severe. } \\
16 \text { divers with previous or } \\
\text { present back pain (first episode } \\
\text { at age } 15 \pm 1.6 \text { years) } \\
\text { Causes of back pain: } \\
\text { - Diving (8) } \\
\text { - Trauma (2) } \\
\text { - Do not know (4) } \\
\text { - Not answered (2) } \\
\text { Pain deteriorated with training } \\
\text { (10) } \\
\text { Pain improved with training (2) }\end{array}$ & $\begin{array}{l}\text { - MRI examination } \\
\text { - Neurological } \\
\text { examination of } \\
\text { lower extremities } \\
\text { and the spine } \\
\text { - Back pain } \\
\text { questionnaire }\end{array}$ & $\begin{array}{l}\text { - MRI abnormalities were observed in } 3 \text { divers at } \\
\text { baseline } \\
\text { - MRI abnormalities were observed in } 4 / 7 \text { divers } \\
\text { trained more than } 8 \mathrm{~h} / \text { week (at follow-up) } \\
\text { - MRI abnormalities were also found in } 8 / 11 \\
\text { divers trained less than } 8 \mathrm{~h} / \text { week } \\
\text { - High prevalence of back pain and MRI changes } \\
\text { was observed; their causal relationship was } \\
\text { confirmed } \\
\text { - Young age was associated with high frequency } \\
\text { of back pain } \\
\text { - Growth spurt was associated with high risk of } \\
\text { occurrence of degenerative changes of the } \\
\text { thoracolumbar spine } \\
\text { - Mild structural scoliosis in the thoracic spine in } \\
1 \text { diver } \\
\text { - Tenderness in the thoracic in } 8 \text { divers } \\
\text { - Tenderness in the lumbar spine in } 5 \text { divers } \\
\text { - } 5 \text { divers presented MRI abnormalities and } \\
\text { tenderness in the thoracolumbar spine } \\
\text { - MRI abnormalities but no tenderness in } 5 \text { divers } \\
\text { - } 5 \text { divers presented tenderness but no MRI } \\
\text { abnormalities in the spine } \\
\text { - MRI abnormalities or tenderness in } 3 \text { divers } \\
\text { - Abnormalities in the thoracolumbar spine at } \\
\text { baseline MRI examination in } 12 \text { out of } 18 \text { divers } \\
\text { - Abnormalities in the thoracolumbar spine at } \\
\text { follow-up MRI examination in } 12 \text { out of } 17 \\
\text { divers } \\
\text { - } 20 \text { new abnormalities in the spine were } \\
\text { observed at follow-up }\end{array}$ \\
\hline $\begin{array}{l}\text { Jonasson et al., } \\
2011\end{array}$ & $\begin{array}{l}\text { - } 75 \text { Swedish top } \\
\text { male athletes (17 } \\
\text { divers, } 17 \\
\text { weight-lifters, } 11 \\
\text { wrestlers, } 13 \\
\text { orienteers, and } 17 \\
\text { ice-hockey players) } \\
\text { - Control group } \\
\text { consisted of } 21 \\
\text { non-athletes }\end{array}$ & $\begin{array}{l}\text { The median age of top } \\
\text { athletes: } 21.5 \text { years } \\
\text { The median age of } \\
\text { non-athletes: } 28 \text { years } \\
\text { The median age of } \\
\text { divers: } 17 \text { years } \\
\text { (youngest group) }\end{array}$ & $\begin{array}{l}\text { The median training days/week } \\
\text { for divers: } 3.5 \\
\text { Training hours/week: } \\
6.5 \pm 2.9 \mathrm{~h} \\
\text { Mean training duration: } \\
8.8 \mathrm{~h} / \text { week } \\
\text { Four divers trained } 11 \mathrm{~h} / \text { week } \\
\text { Training intensity was not } \\
\text { specified ins other group of } \\
\text { athletes. }\end{array}$ & $\begin{array}{l}\text { All athletes and controls with no } \\
\text { knowledge of previous or } \\
\text { present back symptoms }\end{array}$ & $\begin{array}{l}\text { - Self-assessed } \\
\text { pain-oriented } \\
\text { questionnaire } \\
\text { - The EuroQol } \\
\text { questionnaire } \\
\text { - The Oswestry } \\
\text { Disability } \\
\text { Questionnaire }\end{array}$ & $\begin{array}{l}\text { - The frequency of cervical, thoracic and lumbar } \\
\text { pain in the last week/last year was 35/55\%, } \\
22 / 33 \% \text {, and } 50 / 68 \% \text {, respectively }\end{array}$ \\
\hline
\end{tabular}


TABLE 1 | Continued

\begin{tabular}{|c|c|c|c|c|c|c|}
\hline Authors & $\begin{array}{l}\text { Sport and } \\
\text { performance } \\
\text { level }\end{array}$ & Subjects & Training & Back pain and/or injury & $\begin{array}{l}\text { Diagnostics of } \\
\text { back pain and/or } \\
\text { injury }\end{array}$ & Main findings \\
\hline $\begin{array}{l}\text { Kaneoka et al., } \\
2007\end{array}$ & $\begin{array}{l}\text { - } 56 \text { Japanese elite } \\
\text { swimmers ( } 35 \text { male } \\
\text { and } 21 \text { female) - } \\
\text { defined as a } \\
\text { "high-load group" } \\
\text { - } 38 \text { students ( } 24 \\
\text { male and } 14 \\
\text { female) defined as } \\
\text { a "low-load group" } \\
\text { or control group. }\end{array}$ & Age: $15-27$ years & $\begin{array}{l}\text { High load group } \\
\text { Swimming duration: } 9.3 \text { years } \\
\text { Swimming distance: } \\
49,047 \mathrm{~m} / \text { week } \\
\text { Low load group } \\
\text { Swimming duration: } \\
\text { 5.4 years } \\
\text { Swimming distance: } \\
8440 \mathrm{~m} / \text { week }\end{array}$ & $\begin{array}{l}\text { - Low back pain in a high load } \\
\text { group (yes/no): } 43 / 13 \\
\text { - LBP in a low load group } \\
\text { (yes/no): } 33 / 5 \\
\text { - Severe LBP in a high load } \\
\text { group (yes/no): } 29 / 27 \\
\text { - Severe LBP in a low load group } \\
\text { (yes/no): } 14 / 24\end{array}$ & $\begin{array}{l}\text { - Questionnaire } \\
\text { - MRI examination }\end{array}$ & $\begin{array}{l}\text { - Degenerated disks at various disk levels } \\
\text { observed in } 38 \text { elite swimmers and } 11 \text { controls } \\
\text { - The prevalence was significantly greater in elite } \\
\text { swimmers } \\
\text { - No association was observed between the low } \\
\text { back pain, swimming strokes, and disk } \\
\text { degeneration } \\
\text { - L5-S1 intervertebral segment is the most } \\
\text { affected by excessive competitive swimming } \\
\text { activities }\end{array}$ \\
\hline $\begin{array}{l}\text { Schultz and } \\
\text { Gordon, } 2010\end{array}$ & $\begin{array}{l}\text { - } 66 \text { recreational } \\
\text { Australian cyclists } \\
\text { aged } 18 \text { years and } \\
\text { over ( } 49 \text { male and } \\
17 \text { female) } \\
\text { - Divided into LBP } \\
\text { group ( } n=33 \text { ) and } \\
\text { NLBP group } \\
(n=33)\end{array}$ & & $\begin{array}{l}\text { LBP group } \\
\text { Cycling experience: } \\
6.0 \pm 11.6 \text { years } \\
\text { Distance cycled: } \\
250 \pm 131.0 \mathrm{~km} / \text { week } \\
\text { Cycling frequency: } \\
5.0 \pm 1.4 \text { days/week } \\
\text { NLBP group } \\
\text { Cycling experience: } \\
3.0 \pm 7.4 \text { years } \\
\text { Distance cycled: } \\
150.0 \pm 135 \mathrm{~km} / \text { week } \\
\text { Cycling frequency: } \\
4.0 \pm 1.8 \text { days/week }\end{array}$ & $\begin{array}{l}33 \text { cyclists ( } 23 \text { males and } 10 \\
\text { females) reported LBP during } \\
\text { or after cycling within the last } \\
6 \text { months } \\
27 \text { cyclists ( } 26 \text { males and } 7 \\
\text { females) reported no low back } \\
\text { pain during or after cycling }\end{array}$ & $\begin{array}{l}\text { - Survey by Wilber } \\
\text { et al. (1995) }\end{array}$ & $\begin{array}{l}\text { - LBP group cycled significantly more in a week } \\
\text { than NLBP group } \\
\text { - Training volume of } 160 \mathrm{~km} / \text { week or more was } \\
\text { associated with } 3.6 \text { times higher incidence of } \\
\text { LBP than in cyclists with training volume less } \\
\text { than } 160 \mathrm{~km} / \text { week } \\
\text { - Training volume less than } 160 \mathrm{~km} / \text { week } \\
\text { reduced the risk of new occurrence of LBP in } \\
\text { recreational cyclists }\end{array}$ \\
\hline Muyor et al., 2011 & $\begin{array}{l}\text { - } 60 \text { Spanish elite } \\
\text { male cyclists and } \\
60 \text { master cyclists }\end{array}$ & $\begin{array}{l}\text { Elite } \\
\text { Mean age: } \\
22.95 \pm 3.38 \text { years } \\
\text { Master } \\
\text { Mean age: } \\
34.27 \pm 3.05 \text { years }\end{array}$ & $\begin{array}{l}\text { Elite } \\
\text { Training days/week: } 5.5 \pm 1.46 \\
\text { Training hours/day: } 3.23 \pm 0.69 \\
\text { Master } \\
\text { Training days/week: } \\
3.23 \pm 1.35 \\
\text { Training hours/day: } 3.06 \pm 1.28\end{array}$ & $\begin{array}{l}\text { Without spinal pain in } 3 \text { months } \\
\text { prior study enrollment }\end{array}$ & - Spinal Mouse & $\begin{array}{l}\text { - Elite cyclists present thoracic hyperkyphotic } \\
\text { postures in standing position; these changes in } \\
\text { spinal curvatures may be a reason for the } \\
\text { development of LBP }\end{array}$ \\
\hline
\end{tabular}


TABLE 1 | Continued

\begin{tabular}{|c|c|c|c|c|c|c|}
\hline Authors & $\begin{array}{l}\text { Sport and } \\
\text { performance } \\
\text { level }\end{array}$ & Subjects & Training & Back pain and/or injury & $\begin{array}{l}\text { Diagnostics of } \\
\text { back pain and/or } \\
\text { injury }\end{array}$ & Main findings \\
\hline $\begin{array}{l}\text { Ruckstuhl and } \\
\text { Clénin, } 2019\end{array}$ & $\begin{array}{l}\text { - } 111 \text { Swiss elite } \\
\text { cyclists (66 cyclists } \\
\text { of endurance } \\
\text { disciplines and } 45 \\
\text { cyclists of technical } \\
\text { disciplines) } \\
\text { - Endurance athletes } \\
\text { (road cycling, MTB } \\
\text { or cyclo-cross): } 39 \\
\text { males and } 27 \\
\text { females } \\
\text { - Technical discipline } \\
\text { athletes (BMX, trial, } \\
\text { downhill or } 4 \times \text { ): } 38 \\
\text { males and } 7 \\
\text { females } \\
\text { - All cyclists - a } \\
\text { Swiss national } \\
\text { team members }\end{array}$ & $\begin{array}{l}\text { Age of endurance } \\
\text { athletes: } \\
19.5 \pm 5.8 \text { years } \\
\text { Age of technical } \\
\text { athletes: } \\
19.6 \pm 3.5 \text { years }\end{array}$ & Training hours/week: $11.5 \pm 5$ & & $\begin{array}{l}\text { - Core strength } \\
\text { testing } \\
\text { - Back pain } \\
\text { questionnaire } \\
\text { (modified } \\
\text { Oswestry-Disability- } \\
\text { Index) } \\
\text { - Questionnaire on } \\
\text { prior to training in } \\
\text { general and core } \\
\text { strength training } \\
\text { - Sports medical } \\
\text { examination } \\
\text { - Evaluation of the } \\
\text { sitting position on } \\
\text { the bicycle }\end{array}$ & $\begin{array}{l}\text { - Back pain in cyclists is frequent } \\
\text { - In training - every 3rd suffers slight and every } \\
10 \text { th rider moderate to heavy back pain } \\
\text { - In competition - half of the cyclists are affected } \\
\text { by back pain episodes (22.5\% of them have } \\
\text { moderate to heavy back pain) } \\
\text { - } 1 / 3 \text { of elite cyclists have insufficient core } \\
\text { strength } \\
\text { - Core strength of the dorsal muscle chain is } \\
\text { positively related to a lesser frequency of back } \\
\text { pain } \\
\text { - No significant association between back pain } \\
\text { and core strength of the ventral and lateral } \\
\text { chain } \\
-21.6 \% \text { cyclists are free of pain } \\
-31.5 \% \text { of cyclists suffer at least once a month } \\
\text { of back pain } \\
-37.9 \% \text { of cyclists have back pain episodes } \\
2-7 \text { days/month } \\
-9 \% \text { of cyclists suffer from back pain more often } \\
-6 \% \text { of cyclists suffer from back pain nearly } \\
\text { every day } \\
-34.2 \% \text { of cyclists suffer from slight back pain in } \\
\text { training } \\
-9.9 \% \text { of cyclists suffer from moderate to very } \\
\text { heavy back pain in training } \\
-27.9 \% \text { of cyclists suffer from slight back pain in } \\
\text { competition } \\
-22.5 \% \text { of cyclists suffer from moderate to very } \\
\text { heavy back pain in competition } \\
-4.5 \% \text { of athletes are moderately to heavily } \\
\text { limited in daily life due to back pain } \\
- \text { Endurance disciplines athletes suffer from back } \\
\text { pain more than technical disciplines athletes }\end{array}$ \\
\hline $\begin{array}{l}\text { Piotrowska et al., } \\
2017\end{array}$ & $\begin{array}{l}-167 \text { amateur Polish } \\
\text { male cyclists }\end{array}$ & $\begin{array}{l}\text { Age: } 31.0 \pm 8.0 \text { years } \\
\text { Weight: } 74.9 \pm 9.5 \mathrm{~kg} \\
\text { Height: } 1.8 \pm 0.1 \mathrm{~m}\end{array}$ & $\begin{array}{l}\text { Training experience: } \\
7.2 \pm 6.7 \text { years } \\
\text { Training hours/week: } \\
11.8 \pm 6.9\end{array}$ & $\begin{array}{l}\text { Spine pain - experienced by } \\
70 \text { cyclists } \\
\text { Lumbar spine pain (33\%) - one } \\
\text { of the most common pains }\end{array}$ & - Questionnaire & $\begin{array}{l}\text { - Spine pain in } 41 \% \text { of athletes ( } 26 \% \text { is related to } \\
\text { the lumbar spine) } \\
\text { - Number of hours devoted to training/week } \\
\text { influence the case of spine pain }\end{array}$ \\
\hline
\end{tabular}


TABLE 1 | Continued

\begin{tabular}{|c|c|c|c|c|c|c|}
\hline Authors & $\begin{array}{l}\text { Sport and } \\
\text { performance } \\
\text { level }\end{array}$ & Subjects & Training & Back pain and/or injury & $\begin{array}{l}\text { Diagnostics of } \\
\text { back pain and/or } \\
\text { injury }\end{array}$ & Main findings \\
\hline Murray et al., 2009 & $\begin{array}{l}\text { - } 64 \text { English amateur } \\
\text { golfers ( } 43 \text { men } \\
\text { and } 21 \text { women) }\end{array}$ & $\begin{array}{l}\text { LBP group } \\
\text { Age: } 56.4 \pm 8.4 \text { years } \\
\text { Height: } 179.1 \pm 8.7 \mathrm{~cm} \\
\text { Weight: } 85.2 \pm 14.5 \mathrm{~kg} \\
\text { Male to female: } 26: 2 \\
\text { Control group } \\
\text { Age: } 54.3 \pm 14.4 \text { years } \\
\text { Height: } 176.8 \pm 7.8 \mathrm{~cm} \\
\text { Weight: } 78.2 \pm 10.2 \mathrm{~kg} \\
\text { Male to female: } 32: 4\end{array}$ & $\begin{array}{l}\text { LBP group } \\
\text { Rounds/week: } 2.0 \pm 0.9 \\
\text { Years of play: } 27.3 \pm 11.3 \\
\text { Control group } \\
\text { Rounds/week: } 2.1 \pm 1.1 \\
\text { Years of play: } 22.9 \pm 15.6\end{array}$ & $\begin{array}{l}\text { Only golfers with LBP due to } \\
\text { repeated injuries. } \\
\text { LBP group ( } n=28 \text { ) - golfers } \\
\text { with history of LBP within the } \\
\text { last } 12 \text { months prior to the } \\
\text { study enrollment (also currently } \\
\text { suffering from LBP) } \\
\text { Control group ( } n=36 \text { ) - golfers } \\
\text { with no history of LBP }\end{array}$ & $\begin{array}{l}\text { - Questionnaire } \\
\text { including } \\
\text { self-reported LBP in } \\
\text { the past } 12 \text { months } \\
\text { - Body chart }\end{array}$ & $\begin{array}{l}\text { - The weak causal relationship between LBP and } \\
\text { hip rotation }\end{array}$ \\
\hline $\begin{array}{l}\text { McHardy et al., } \\
2007\end{array}$ & $\begin{array}{l}\text { - } 588 \text { Australian } \\
\text { amateur golfers } \\
\text { (473 men and } 115 \\
\text { women) }\end{array}$ & $\begin{array}{l}\text { All } \\
\text { Age: } 59.1 \pm 12.9 \text { years } \\
\text { Men } \\
\text { Age: } 58.7 \pm 13.5 \text { years } \\
\text { Women } \\
\text { Age: } 60.8 \pm 9.9 \text { years }\end{array}$ & $\begin{array}{l}\text { Men } \\
\text { Years of play: } 31.8 \pm 15.3 \\
\text { Rounds/week: } 1.7 \pm 1.1 \\
\text { Women } \\
\text { Years of play: } 21.7 \pm 14.6 \\
\text { Rounds/week: } 1.8 \pm 1.1\end{array}$ & & $\begin{array}{l}\text { - A prospective } \\
\text { survey } \\
\text { questionnaire }\end{array}$ & $\begin{array}{l}\text { - The observed annual injury rate was } 15.8 \\
\text { injuries/100 players }(0.4-0.6 \\
\text { injuries/1000 h/person) } \\
\text { - Men report higher rate of injuries/100 players } \\
\text { than women } \\
\text { - The lower back is the most frequently injured } \\
\text { part (18.3\%) as a result of the golf swing }\end{array}$ \\
\hline $\begin{array}{l}\text { Saraceni et al., } \\
2017\end{array}$ & $\begin{array}{l}-16 \text { recreational } \\
\text { male golfers } \\
\text { aged } \geq 18 \text { years }\end{array}$ & $\begin{array}{l}\text { Played or practiced golf } \\
\text { for an average of } \\
\geq 4 \mathrm{~h} / \text { week over the } \\
\text { past } 2 \text { years }\end{array}$ & $\begin{array}{l}\text { LBP group } \\
\text { Years of play: } 19 \pm 9 \\
\text { Weekly golfing time: } 19 \pm 16 \mathrm{~h} \\
\text { No pain group } \\
\text { Years of play: } 14 \pm 6 \\
\text { Weekly golfing time: } 23 \pm 15 \mathrm{~h}\end{array}$ & $\begin{array}{l}\text { LBP group } \\
\text { - LBP over } 2 \text { years preceding the } \\
\text { data collection } \\
\text { - } 2 \text { or more episodes of LBP } \\
\text { - Aggravated by golf } \\
\text { - Prevented golfers from playing } \\
\text { in } 24 \text { months before data } \\
\text { collection } \\
\text { - Most recent episode occurred } \\
\text { in } 6 \text { months prior to study }\end{array}$ & $\begin{array}{l}\text { - Clinical } \\
\text { examination - } \\
\text { motion analysis }\end{array}$ & $\begin{array}{l}\text { - Reduced lead hip internal rotation in the LBP } \\
\text { group compared to no pain group during prone } \\
\text { passive and standing clinical measures }\end{array}$ \\
\hline
\end{tabular}


TABLE 1 | Continued

Dennis et al., 2005 - 44 Australian male fast bowlers

compete at club

and district levels

Piazza et al., 2009

60 former elite

women gymnasts

from Italian nationa

team

- International,

national level and

Olympic Games

competitors

Tertti et al., $1990 \quad-35$ competitive gymnasts (18 male and 17 female)

- District level gymnast $(n=12)$

- National level

gymnasts $(n=21)$

International leve

gymnasts $(n=2)$
Subjects

Training

Back pain and/or injury

Diagnostics of

Main findings

back pain and/o

injury

Division I $(n=119) \quad$ Division

Age: $23.5 \pm 3.4$ years

Golf career (years): $12.2 \pm 2.6$

Height: $166.1 \pm 5.3 \mathrm{~cm}$ Tournament games/year: 31

Weight: $60.8 \pm 5.6 \mathrm{~kg} \quad$ (20 last for 3 days and 11 for

Division II $(n=121)$

Age: $22.4 \pm 2.9$ years

Height: $165 \pm 5.2 \mathrm{~cm}$

Weight: $59.7 \pm 6.0 \mathrm{~kg}$

Division III $(n=123)$

Age: $21.0 \pm 3.2$ years

Height: $165 \pm 5.4 \mathrm{~cm}$

Weight: $58.7 \pm 8.0 \mathrm{~kg}$

4 days)

Hours to complete each

round/year: 520

Number of training

hours/competition day: 6

Division II and III

Golf career (years):

$10 \pm 2.8$ and $7.7 \pm 2.7$,

respectively

Tournament games/year: 19

and 16 (2-day tournament

games)

Hours to complete each

round/year: 190 and 160

Number of training

hours/competition day: 7 and 7.5

Age: $14.7 \pm 1.4$ years $\quad$ Bowling days in total $(44$

bowlers): 1783 (696 match

days and 1087 training days)

Match/day: 1

Training days/week: 1.5

Age: $38 \pm 7$ years

Weight: $54.5 \pm 5 \mathrm{~kg}$

Training intensity:

$29.7 \pm 11.4 \mathrm{~h} /$ week

Height: $1.66 \pm 0.44 \mathrm{~m}$

Age: $12 \pm 2.6$ years

Weight: $39 \pm 9.6 \mathrm{~kg}$

Height: $150 \pm 13 \mathrm{~cm}$
$25 \%$ bowlers reported an overuse injury during the

\section{2 gymnasts with LBP}

Mean age of symptoms onset:

$19 \pm 8$ years

LBP during activity in $71.4 \%$ of

gymnasts
- Practice time: $12 \pm 5.5 \mathrm{~h} /$ week

- Years of practice: $4.2 \pm 2.5$
Interview with physiotherapist MRI examination

- Interview

- Self-administered questionnaire

- Questionnaire

- Clinical examination

$-\mathrm{MRI}$ and

radiographs

- YISSEM ISS survey - A total of 510 injuries were recorded

- 174 injuries occurred in Division I, 166 in

Division II, and 170 in Division III

- The most frequent mechanism for injury was

the golf swing upon ball impact

- The injury risk in female golfers was associated

with number of competitions

- Lumbar spine/lower back is one of the most common iniured part

An increased risk of injury was observed for those who bowled more than 2.5 days/week or for those with more than 50 deliveries per day $-25 \%$ bowlers reported an overuse injury, seven of them sustaining a back injury

- Occurrence of back pain during the sport activity was associated with an early onset of symptoms of LBP after the retire from competitions

- 11 gymnasts suffer from episodes of LBP during exercises

-8 gymnasts have evidence of back trauma - 3 out of 35 gymnasts have MRI evidence of degenerated discs 
TABLE 1 | Continued

\begin{tabular}{|c|c|c|c|c|c|c|}
\hline Authors & $\begin{array}{l}\text { Sport and } \\
\text { performance } \\
\text { level }\end{array}$ & Subjects & Training & Back pain and/or injury & $\begin{array}{l}\text { Diagnostics of } \\
\text { back pain and/or } \\
\text { injury }\end{array}$ & Main findings \\
\hline $\begin{array}{l}\text { Goldstein et al., } \\
1991\end{array}$ & $\begin{array}{l}\text { - } 33 \text { top level female } \\
\text { gymnasts (11 } \\
\text { pre-elite, } 14 \text { elite, } \\
\text { national, and } 8 \\
\text { Olympic levels) } \\
\text { regardless of back } \\
\text { pain or injury } \\
\text { - Control group: } \\
\text { female swimmers }\end{array}$ & $\begin{array}{l}\text { Top gymnasts } \\
\text { Pre-elite gymnasts } \\
\text { Mean age: } 11.8 \text { years } \\
\text { Elite gymnasts } \\
\text { Mean age: } 16.6 \text { years } \\
\text { Older national/Olympic } \\
\text { gymnasts } \\
\text { Average age: } \\
25.7 \text { years } \\
\text { Controls } \\
\text { The AA and AAA } \\
\text { swimmers } \\
\text { Average age: } \\
14.6 \text { years } \\
\text { Q and national ranked } \\
\text { swimmers } \\
\text { Average age: } \\
18.6 \text { years }\end{array}$ & $\begin{array}{l}\text { Top gymnasts } \\
\text { Pre-elite gymnasts } \\
\text { Training h/week: } 18.2 \text { in their } \\
\text { most current year } \\
\text { Elite gymnasts } \\
\text { Training h/week: } 23.2 \text { in their } \\
\text { most current year } \\
\text { Older/Olympic gymnasts } \\
\text { Training h/week: } 22.1 \text { in their } \\
\text { most current year } \\
\text { Controls } \\
\text { The AA and AAA swimmers } \\
\text { Training h/week: } 11.3 \text { in their } \\
\text { most current year } \\
\text { Q and national ranked } \\
\text { swimmers } \\
\text { Training h/week: } 16.4 \text { in their } \\
\text { most current year }\end{array}$ & No initial signs of LBP & - MRI examination & $\begin{array}{l}\text { - } 1 \text { pre-elite, } 6 \text { elite and } 5 \text { Olympic level gymnasts } \\
\text { have spine abnormalities } \\
\text { - } 15.8 \% \text { of swimmers (control group) have spine } \\
\text { abnormalities } \\
\text { - Training volume per week and age are } \\
\text { associated with MRI abnormalities } \\
\text { - Top female gymnasts are more prone to spine } \\
\text { injuries }\end{array}$ \\
\hline Reis et al., 2015 & $\begin{array}{l}-72 \text { Brazilian jiu-jitsu } \\
\text { athletes ( } 36 \\
\text { professional and } 36 \\
\text { recreational level } \\
\text { athletes) }\end{array}$ & Median age: 25.5 years & $\begin{array}{l}\text { Median training experience: } \\
8 \text { years } \\
\text { Training frequency: } 2-7 \\
\text { times/week } \\
\text { Median duration of each } \\
\text { practice: } 2 \mathrm{~h} / \text { day }\end{array}$ & $\begin{array}{l}\text { Athletes without history of LBP } \\
\text { before training participation }\end{array}$ & $\begin{array}{l}\text { - Questionnaire } \\
\text { - Quebec Back Pain } \\
\text { Disability Scale } \\
\text { (QBPDS) }\end{array}$ & $\begin{array}{l}\text { - Chronic LBP in } 58 \text { of jiu jitsu athletes (32 } \\
\text { professional and } 26 \text { recreational) } \\
\text { - High prevalence of LBP in jiu-jitsu athletes } \\
\text { - Professional jiu-jitsu athletes are more prone to } \\
\text { developing of chronic LBP }\end{array}$ \\
\hline Almeida et al., 2012 & $\begin{array}{l}\text { - } 42 \text { judo athletes ( } 22 \\
\text { male and } 20 \\
\text { female) }\end{array}$ & $\begin{array}{l}\text { LBP group } \\
\text { Age: } 16.7 \pm 2.9 \text { years } \\
\text { Weight: } 60.1 \pm 1.34 \mathrm{~kg} \\
\text { Height: } 1.64 \pm 0.1 \mathrm{~m} \\
\text { Control group } \\
\text { Age: } 16.3 \pm 2 \text { years } \\
\text { Weight: } 61.5 \pm 9.9 \mathrm{~kg} \\
\text { Height: } 1.65 \pm 0.1 \mathrm{~m}\end{array}$ & $\begin{array}{l}\text { LBP group } \\
\text { Training experience: } \\
8.9 \pm 2.9 \text { years } \\
\text { Weekly practice frequency: } \\
5 \pm 0.8 \\
\text { Hours of practice/day: } \\
2.1 \pm 0.4 \\
\text { Hours of practice/week: } \\
10.1 \pm 1.3 \\
\text { Control group } \\
\text { Training experience: } \\
8.4 \pm 3.1 \text { years } \\
\text { Weekly practice frequency: } \\
4.8 \pm 0.7 \\
\text { Hours of practice/day: } \\
2.1 \pm 0.5 \\
\text { Hours of practice/week } \\
10.5 \pm 2.8\end{array}$ & $\begin{array}{l}\text { Years with low back pain: } \\
1.2 \pm 0.5 \\
\text { Number of episodes in previous } \\
12 \text { months: } 4.8 \pm 8.3 \\
\text { Mean score on visual analog } \\
\text { pain scale: } 4.1 \pm 2.6\end{array}$ & $\begin{array}{l}\text { - Computed } \\
\text { photogrammetry } \\
\text { - Pain scale }\end{array}$ & $\begin{array}{l}\text { - Deficits in hip rotation and greater asymmetry } \\
\text { between limbs were observed in judo athletes } \\
\text { with a history of LBP }\end{array}$ \\
\hline
\end{tabular}


TABLE 1 | Continued

\begin{tabular}{|c|c|c|c|c|c|c|}
\hline Authors & $\begin{array}{l}\text { Sport and } \\
\text { performance } \\
\text { level }\end{array}$ & Subjects & Training & Back pain and/or injury & $\begin{array}{l}\text { Diagnostics of } \\
\text { back pain and/or } \\
\text { injury }\end{array}$ & Main findings \\
\hline $\begin{array}{l}\text { Rostami et al., } \\
2015\end{array}$ & $\begin{array}{l}\text { - } 14 \text { professional } \\
\text { off-road male } \\
\text { cyclists } \\
\text { - } 24 \text { controls } \\
\text { - Cyclists competed } \\
\text { at the national and } \\
\text { international level } \\
\text { during the past } \\
12 \text { months }\end{array}$ & $\begin{array}{l}\text { Off-road cyclists } \\
\text { Age: } 27.2 \pm 4.74 \text { years } \\
\text { Height: } 172.7 \pm 5.5 \mathrm{~cm} \\
\text { Weight: } 71.5 \pm 11.9 \mathrm{~kg} \\
\text { Controls } \\
\text { Age: } 27.8 \pm 5.26 \text { years } \\
\text { Height: } \\
179.7 \pm 5.66 \mathrm{~cm} \\
\text { Weight: } 80.2 \pm 10.5 \mathrm{~kg}\end{array}$ & $\begin{array}{l}\text { Off-road cyclists } \\
\text { Cycling distance/week: } \\
236.1 \pm 116.16 \mathrm{~km} \\
\text { Road cycling distance/week: } \\
165.0 \pm 88.82 \mathrm{~km} \\
\text { Off-road cycling distance/week: } \\
71.1 \pm 40.96 \mathrm{~km} \\
\text { Controls } \\
\text { Cycling distance/week: } \\
299.6 \pm 169.13 \mathrm{~km} \\
\text { Road cycling distance/week: } \\
240.4 \pm 161.82 \mathrm{~km} \\
\text { Off-road cycling distance/week: } \\
58.7 \pm 20.50 \mathrm{~km}\end{array}$ & $\begin{array}{l}\text { Cyclists with bilateral } \\
\text { non-specific LBP for more than } \\
12 \text { months } \\
\text { LBP was defined as pain in the } \\
\text { area between the } 12 \text { th rib and } \\
\text { the inferior gluteal fold } \\
\text { responsible for a limitation in } \\
\text { the performing of usual daily } \\
\text { activities for more than } 1 \text { day } \\
\text { during the past } 4 \text { weeks }\end{array}$ & $\begin{array}{l}\text { - Ultrasonic } \\
\text { measurements } \\
\text { - Flexibility } \\
\text { assessment } \\
\text { - Dynamometry }\end{array}$ & $\begin{array}{l}\text { - Lower thickness of transversus abdominis and } \\
\text { cross sectional area of lumbar multifidus spinae } \\
\text { in cyclists with LBP compared to controls }\end{array}$ \\
\hline $\begin{array}{l}\text { Trompeter et al., } \\
2019\end{array}$ & $\begin{array}{l}\text { - } 156 \text { German } \\
\text { rowers (104 elite } \\
\text { and } 52 \text { non-elite / } \\
49 \text { sculls, and } 76 \\
\text { sweeps) } \\
\text { - } 90 \text { males and } 65 \\
\text { females } \\
\text { - } 166 \text { physically } \\
\text { active non-rowing } \\
\text { controls }\end{array}$ & $\begin{array}{l}\text { Rowers } \\
\text { Age: } 22.2 \pm 5.1 \text { years } \\
\text { Height: } 183.4 \pm 8.3 \mathrm{~cm} \\
\text { Weight: } 78.1 \pm 11.5 \mathrm{~kg} \\
\text { Sex (m/f): } 57.1 / 41.7 \% \\
\text { Controls } \\
\text { Age: } 21.2 \pm 2.0 \text { years } \\
\text { Height: } 180.0 \pm 8.9 \mathrm{~cm} \\
\text { Weight: } 74.0 \pm 10.3 \mathrm{~kg} \\
\text { Sex (m/f): } 74.7 / 24.1 \%\end{array}$ & $\begin{array}{l}\text { Rowers } \\
\text { Training volume: } \\
16.3 \pm 8.2 \mathrm{~h} / \text { week } \\
\text { Years of rowing training: } \\
9.5 \pm 5.4 \\
\text { Controls } \\
\text { Training volume: } \\
10.8 \pm 5.0 \mathrm{~h} / \text { week }\end{array}$ & & $\begin{array}{l}\text { - Back pain } \\
\text { questionnaire } \\
\text { - Chronic Pain Grade } \\
\text { (CPG) }\end{array}$ & $\begin{array}{l}\text { - High back pain prevalence and severity were } \\
\text { observed in rowers than controls, and among } \\
\text { scull than sweep rowers } \\
\text { - The lower back was the most frequent area for } \\
\text { pain occurrence in rowers of all competition } \\
\text { levels and also in controls } \\
\text { - Age, sex, training volume, kinematics, strength, } \\
\text { and ergometer training were associated with } \\
\text { higher prevalence of back pain in rowers }\end{array}$ \\
\hline $\begin{array}{l}\text { Alricsson et al., } \\
2016\end{array}$ & $\begin{array}{l}\text { - } 51 \text { elite Swedish } \\
\text { cross-country } \\
\text { skiers }\end{array}$ & $\begin{array}{l}\text { Age: } 16-19 \text { years } \\
\text { Sex }(m / f): 30 / 21\end{array}$ & $\begin{array}{l}\text { Training hours/week: } \\
\text { Pre-season } \\
11.3 \pm 2.6 \\
\text { Season } \\
9.0 \pm 1.6 \\
\text { Number of } \\
\text { competitions/season: } \\
16.5 \pm 5.9 \\
\text { Trained } \\
13.8 \pm 1.9(7-16) \text { years }\end{array}$ & $\begin{array}{l}\text { LBP - defined according to } \\
\text { European Guidelines } \\
\text { (Airaksinen et al., 2006) as pain } \\
\text { or discomfort somewhere } \\
\text { between the } 12 \text { th rib and the } \\
\text { lower gluteal fold }\end{array}$ & $\begin{array}{l}\text { - Questionnaire } \\
\text { about training and } \\
\text { competition status } \\
\text { - Back pain } \\
\text { questionnaire } \\
\text { - DeBrunner's } \\
\text { kyphometer } \\
\text { - Lumbar locked } \\
\text { thoracic rotation } \\
\text { test } \\
\text { - Modified Thomas } \\
\text { test } \\
\text { - Prone active } \\
\text { straight leg rising }\end{array}$ & $\begin{array}{l}\text { - Participants with greater lordosis than kyphosis } \\
\text { suffer from LBP more than those without } \\
\text { kyphosis } \\
\text { - Sagittal spinal alignment are related to LBP in } \\
\text { elite cross-country skiers } \\
\text { - The range of motion of the thoracic spine and } \\
\text { hips do not affect the prevalence of LBP in elite } \\
\text { cross-country skiers }\end{array}$ \\
\hline
\end{tabular}


TABLE 1 | Continued

\begin{tabular}{|c|c|c|c|c|c|c|}
\hline Authors & $\begin{array}{l}\text { Sport and } \\
\text { performance } \\
\text { level }\end{array}$ & Subjects & Training & Back pain and/or injury & $\begin{array}{l}\text { Diagnostics of } \\
\text { back pain and/or } \\
\text { injury }\end{array}$ & Main findings \\
\hline Siewe et al., 2011 & $\begin{array}{l}\text { - } 245 \text { German } \\
\text { competitive and } \\
\text { elite powerlifters } \\
\text { ( } 219 \text { male and } 26 \\
\text { female) } \\
\text { - } 225 \text { powerlifters - } \\
\text { winners with } 154 \\
\text { medals on the } \\
\text { national or } \\
\text { international levels }\end{array}$ & $\begin{array}{l}\text { All powerlifters } \\
\text { Age: } 37.8 \pm 14.3 \text { years } \\
\text { Weight (off-season): } \\
89.1 \pm 18.4 \mathrm{~kg} \\
\text { Male } \\
\text { Weight: } 91.9 \pm 17.1 \mathrm{~kg} \\
\text { Female } \\
\text { Weight: } 65.4 \pm 10.2 \mathrm{~kg}\end{array}$ & $\begin{array}{l}\text { All powerlifters } \\
\text { The maximum average loads } \\
\text { lifted: squat } 205.8 \pm 69.12 \mathrm{~kg} \text {, } \\
\text { bench press } 151.6 \pm 52.4 \mathrm{~kg}, \\
\text { and deadlift } 214.2 \pm 54.8 \mathrm{~kg} \\
\text { Training experience: } \\
13.6 \pm 10.5 \text { years } \\
\text { During the competitive season, } \\
88 \% \text { of subjects worked } 3-7 \\
\text { times/week } \\
\text { The average workout time: } \\
119.1 \pm 39.7 \text { min/day } \\
\text { Male } \\
\text { The maximum average loads } \\
\text { lifted: squat } 204.3 \pm 69.7 \mathrm{~kg}, \\
\text { bench press } 152.0 \pm 52.9 \mathrm{~kg} \\
\text { and deadlift } 213.4 \pm 55.2 \mathrm{~kg} \\
\text { Female } \\
\text { The maximum average loads } \\
\text { lifted: squat } 209.7 \pm 64.7 \mathrm{~kg}, \\
\text { bench press } 148.2 \pm 48.3 \mathrm{~kg}, \\
\text { and deadlift } 221.6 \pm 52.6 \mathrm{~kg}\end{array}$ & & - Questionnaire & $\begin{array}{l}\text { - Injuries of the lumbar spine in } 108 \text { powerlifters } \\
\text { - The most frequent diagnoses are sciatica and } \\
\text { myogelosis } \\
\text { - Gender, exercise weight, workout duration, } \\
\text { competition level, and routine endurance } \\
\text { training have no impact on injury rates } \\
\text { - Using of supporting devices was associated } \\
\text { with more lumbar spine problems }\end{array}$ \\
\hline Junior et al., 2013 & $\begin{array}{l}\text { - } 191 \text { recreational } \\
\text { runners ( } 141 \text { males } \\
\text { and } 50 \text { females) }\end{array}$ & $\begin{array}{l}\text { All } \\
\text { Age: } 42.8 \pm 10.5 \text { years } \\
\text { Height: } 171.1 \pm 9.4 \mathrm{~cm} \\
\text { Weight: } 72.0 \pm 14.0 \mathrm{~kg} \\
\text { Injured } \\
\text { Age: } 41.8 \pm 10.2 \text { years } \\
\text { Height: } 172.4 \pm 8.8 \mathrm{~cm} \\
\text { Weight: } 73.1 \pm 11.8 \mathrm{~kg} \\
\text { Non-injured } \\
\text { Age: } 42.9 \pm 10.5 \text { years } \\
\text { Height: } 170.5 \pm 9.7 \mathrm{~cm} \\
\text { Weight: } 71.4 \pm 14.9 \mathrm{~kg}\end{array}$ & $\begin{array}{l}\text { All } \\
\text { Distance (km/week): } 28.5 \\
\text { (15.0-41.0) } \\
\text { Duration (min/session): } 60 \\
\text { (50-80) } \\
\text { Injured } \\
\text { Distance (km/week): } 15 \\
\text { (2.5-26.3) } \\
\text { Duration (min/session): } 50 \\
\text { (15-60) } \\
\text { Non-injured } \\
\text { Distance (km/week): } 30 \\
\text { (18.0-42.5) } \\
\text { Duration (min/session): } 60 \\
\text { (50-90) }\end{array}$ & $\begin{array}{l}\text { Running related injury was } \\
\text { defined as any pain of } \\
\text { musculoskeletal origin } \\
\text { attributed to running and } \\
\text { associated with absence at } \\
\text { least one training unit (Bovens } \\
\text { et al., 1989; Macera et al., } \\
\text { 1989; van Middelkoop et al., } \\
\text { 2007, 2008) }\end{array}$ & $\begin{array}{l}\text { - 10-point pain } \\
\text { numerical rating } \\
\text { scale } \\
\text { - Survey }\end{array}$ & $\begin{array}{l}\text { - Running related injuries in lumbar spine in } 12 \\
\text { (14\%) runners, duration of LBP: } \\
2.4 \pm 0.8 \text { weeks, pain intensity: } 5.2 \pm 2.5\end{array}$ \\
\hline
\end{tabular}


TABLE 1 | Continued

\begin{tabular}{|c|c|c|c|c|c|c|}
\hline Authors & $\begin{array}{l}\text { Sport and } \\
\text { performance } \\
\text { level }\end{array}$ & Subjects & Training & Back pain and/or injury & $\begin{array}{l}\text { Diagnostics of } \\
\text { back pain and/or } \\
\text { injury }\end{array}$ & Main findings \\
\hline $\begin{array}{l}\text { Villavicencio et al., } \\
2006\end{array}$ & $\begin{array}{l}\text { - } 87 \text { triathletes (31 } \\
\text { male and } 56 \\
\text { female) from } \\
\text { Colorado } \\
\text { - Elite level (6) } \\
\text { - Intermediate level } \\
\text { (65) } \\
\text { - Beginners (16) }\end{array}$ & $\begin{array}{l}\text { All } \\
\text { Age: } 36.1 \text { years } \\
\text { Women } \\
\text { Age: } 34.1 \text { years } \\
\text { Men } \\
\text { Age: } 39.8 \text { years } \\
\text { Triathletes with acute } \\
\text { lumbar pain }(n=37) \\
\text { Mean age: } 36.3 \text { years } \\
\text { Triathletes with } \\
\text { subacute lumbar pain } \\
(n=8) \\
\text { Mean age: } 36.2 \text { years } \\
\text { Triathletes with chronic } \\
\text { lumbar pain }(n=14) \\
\text { Mean age: } 36.9 \text { years }\end{array}$ & $\begin{array}{l}\text { All triathletes } \\
\text { Number of triathlons: } 22.4 \\
\text { Combined training time: } \\
12.5 \mathrm{~h} / \text { week } \\
\text { Swimming, biking, and running } \\
\text { accounted for } 22.47 \text { and } 31 \% \\
\text { of the training time, respectively } \\
\text { Triathletes with acute lumbar } \\
\text { pain } \\
\text { Training volume (hours/week): } \\
14.9 \\
\text { Number of running races and } \\
\text { triathlons: } 24.1 \\
\text { Triathletes with subacute } \\
\text { lumbar pain } \\
\text { Training volume (hours/week): } \\
17.3 \\
\text { Number of running races and } \\
\text { triathlons: } 24.2 \\
\text { Triathletes with chronic lumbar } \\
\text { pain } \\
\text { Training volume (hours/week): } \\
15.0 \\
\text { Number of running races and } \\
\text { triathlons: } 21.4\end{array}$ & $\begin{array}{l}\text { The incidence of lumbar } \\
\text { discogenic back pain was } \\
\text { defined according to the } \\
\text { duration of symptoms for the } \\
\text { most recent pain episode } \\
\text { The lifetime incidence of LBP: } \\
67.8 \% \text { ( } 59 \text { out of } 87 \text { triathletes) } \\
\text { LBP related to sports injuries: in } \\
48 \text { (81.4\%) out of } 59 \text { triathletes } \\
\text { Triathletes with acute lumbar } \\
\text { pain } \\
\text { - LBP lasted fewer than } 7 \text { days } \\
\text { - Triathletes with subacute } \\
\text { lumbar pain } \\
\text { - Most recent back pain episode } \\
\text { lasted from } 7 \text { days to } 3 \text { months } \\
\text { - In } 14 \text { out of } 59 \text { triathletes, the } \\
\text { most recent LBP episode } \\
\text { lasted more than } 3 \text { months } \\
\text { - Number of sports-related } \\
\text { injuries: } 1.5 \\
\text { - Triathletes with chronic lumbar } \\
\text { pain } \\
\text { - The most recent LBP episode } \\
\text { lasted more than } 3 \text { months } \\
\text { - } 9 \text { triathletes noted sciatica } \\
\text { symptoms }\end{array}$ & $\begin{array}{l}\text { - On-line } \\
\text { questionnaire }\end{array}$ & $\begin{array}{l}\text { - No significant association between LBP and } \\
\text { age, athletic status, or training duration } \\
\text { - A strong tendency toward LBP in triathletes } \\
\text { with years of experience } \\
\text { - Strong association was observed between the } \\
\text { number of injuries and the occurrence of LBP }\end{array}$ \\
\hline Sekine et al., 2014 & $\begin{array}{l}\text { - } 68 \text { Japanese } \\
\text { collegiate rowers } \\
\text { (43 men and } 25 \\
\text { women) } \\
\text { - International } \\
\text { competition level } \\
\text { (15) } \\
\text { - National } \\
\text { competition level } \\
\text { (49) } \\
\text { - Regional } \\
\text { competition level (4) } \\
\text { - Male rowers mainly } \\
\text { participated in } \\
\text { sweep oar rowing, } \\
\text { while female rowers } \\
\text { mainly participated } \\
\text { in scull oar rowing }\end{array}$ & $\begin{array}{l}\text { Men } \\
\text { Age: } 19.4 \pm 1.1 \text { years } \\
\text { Height: } 176.4 \pm 5.9 \mathrm{~cm} \\
\text { Weight: } 74.3 \pm 8.1 \mathrm{~kg} \\
\text { Women } \\
\text { Age: } 19.7 \pm 1.1 \text { years } \\
\text { Height: } 164.2 \pm 4.3 \mathrm{~cm} \\
\text { Weight: } 60.0 \pm 5.4 \mathrm{~kg}\end{array}$ & $\begin{array}{l}\text { Training volume: } \\
11 \text { sessions/week } \\
\text { Training duration: } 2 \mathrm{~h} \\
\text { Career duration: } \\
4.8 \pm 1.4 \text { years (men) } \\
5.1 \pm 2.0 \text { years (women) }\end{array}$ & & $\begin{array}{l}\text { - MRI examination } \\
\text { - Injury surveillance }\end{array}$ & $\begin{array}{l}\text { - } 48.8 \% \text { of male rowers and } 40.0 \% \text { of female } \\
\text { rowers presented disc degeneration } \\
\text { - Progression of disc degeneration after } 2 \text { years } \\
\text { in } 5 \text { rowers } \\
\text { - LBP during } 2 \text { years in } 6 \text { rowers; progression of } \\
\text { disk degeneration in } 4 \text { of them } \\
\text { - The prevalence of disc degeneration in lumbar } \\
\text { spine among rowers was } 45.6 \%\end{array}$ \\
\hline
\end{tabular}


TABLE 1 | Continued

\begin{tabular}{|c|c|c|c|c|c|c|}
\hline Authors & $\begin{array}{l}\text { Sport and } \\
\text { performance } \\
\text { level }\end{array}$ & Subjects & Training & Back pain and/or injury & $\begin{array}{l}\text { Diagnostics of } \\
\text { back pain and/or } \\
\text { injury }\end{array}$ & Main findings \\
\hline Roussel et al., 2013 & $\begin{array}{l}-40 \text { Belgian } \\
\text { pre-professional } \\
\text { dancers ( } 2 \text { males, } \\
38 \text { females) }\end{array}$ & $\begin{array}{l}\text { Age: } 20.3 \pm 2.4 \text { years } \\
\text { Height: } \\
1.66 .2 \pm 0.06 \mathrm{~m} \\
\text { Weight: } \\
56.43 \pm 5.71 \mathrm{~kg}\end{array}$ & $\begin{array}{l}\text { Physical activity during classes: } \\
21.5 \pm 2.1 \mathrm{~h} / \text { week } \\
\text { Physical activity outside } \\
\text { classes: } 4.6 \pm 1.3 \mathrm{~h} / \text { week }\end{array}$ & $\begin{array}{l}41 \% \text { dancers with occurrence } \\
\text { of LBP in the last year }\end{array}$ & $\begin{array}{l}\text { - Questionnaires } \\
\text { (SF-36, } \\
\text { self-established } \\
\text { medical) } \\
\text { - The Tampa Scale } \\
\text { for Kinesiophobia } \\
\text { (TSK) } \\
\text { - The Visual } \\
\text { Analogue Scale } \\
\text { (pain severity) } \\
\text { - Clinical test battery }\end{array}$ & $\begin{array}{l}\text { - Dancers with a history of LBP presented poorer } \\
\text { lumbo-pelvic motor control than those without } \\
\text { history of LBP } \\
\text { - Pre-professional dancers suffer from LBP } \\
\text { frequently } \\
\text { - LBP was associated with decreased motor } \\
\text { control in dancers }\end{array}$ \\
\hline $\begin{array}{l}\text { Thoreson et al., } \\
\text { 2017b }\end{array}$ & $\begin{array}{l}\text { - } 16 \text { elite Swedish } \\
\text { Mogul skiers (14 } \\
\text { males and } 2 \\
\text { females) } \\
\text { - } 28 \text { age-matched } \\
\text { non-athletes } \\
\text { (control group) }\end{array}$ & $\begin{array}{l}\text { Mogul skiers } \\
\text { Age: } 17.6 \pm 1.02 \text { years } \\
\text { Height: } 177.0 \pm 6.9 \mathrm{~cm} \\
\text { Weight: } \\
70.8 \pm 10.62 \mathrm{~kg} \\
\text { Control group } \\
\text { Age: } 16.4 \pm 0.57 \text { years } \\
\text { Height: } 172 \pm 8.56 \mathrm{~cm} \\
\text { Weight: } 67 \pm 17.91 \mathrm{~kg}\end{array}$ & $\begin{array}{l}\text { Mogul skiers } \\
12 \text { skiers exercised more than } \\
11 \mathrm{~h} / \text { week } \\
2 \text { skiers exercised } \\
9-11 \mathrm{~h} / \text { week } \\
2 \text { skiers exercised } \\
6-8 \mathrm{~h} / \text { week } \\
\text { Control group } \\
\text { All controls exercised less than } \\
8 \text { h/week } \\
5 \text { controls without exercise } \\
\text { experience }\end{array}$ & $\begin{array}{l}\text { Back pain was defined as any } \\
\text { present or previous pain in the } \\
\text { thoracolumbar back } \\
\text { - It was self-assessed } \\
\text { - It was graded moderate or } \\
\text { severe depends on if daily living } \\
\text { and physical activities were } \\
\text { affected by pain or not }\end{array}$ & $\begin{array}{l}\text { - MRI examination } \\
\text { - Back pain } \\
\text { questionnaires } \\
\text { - Visual Analogue } \\
\text { Scale (the location } \\
\text { and type of pain) }\end{array}$ & $\begin{array}{l}\text { - More MRI abnormalities in Mogul skiers } \\
\text { compared to the controls ( } 7.25 \text { vs. 3.78) } \\
\text { - No difference in LBP lifetime prevalence } \\
\text { between skiers and controls } \\
\text { - No association between disc degeneration and } \\
\text { back pain } \\
\text { - Increased risk of developing spinal } \\
\text { abnormalities in elite Mogul skiers due to the } \\
\text { different high loads than controls }\end{array}$ \\
\hline Witwit et al., 2018 & $\begin{array}{l}\text { - } 75 \text { Swedish elite } \\
\text { skiers (59 alpine } \\
\text { and } 16 \text { mogul } \\
\text { skiers) } \\
\text { - Elite level within the } \\
\text { high school } \\
\text { competitions } \\
\text { - } 27 \text { non-athletic } \\
\text { first-year high } \\
\text { school students } \\
\text { (control group) }\end{array}$ & $\begin{array}{l}\text { Elite skiers } \\
\text { Age: } 18.2 \pm 1.13 \text { years } \\
\text { Height: } 174 \pm 8.2 \mathrm{~cm} \\
\text { Weight: } 70.9 \pm 9.14 \mathrm{~kg} \\
\text { Control group } \\
\text { Age: } 16.4 \pm 0.58 \text { years } \\
\text { Height: } 172 \pm 8.57 \mathrm{~cm} \\
\text { Weight: } 67 \pm 17.90 \mathrm{~kg}\end{array}$ & $\begin{array}{l}\text { Elite skiers } \\
9-11 \text { training hours/week (74\%) } \\
\text { Control group } \\
2-5 \text { training hours/week (78\%) }\end{array}$ & & $\begin{array}{l}\text { - MRI examination } \\
\text { - Back pain } \\
\text { questionnaires }\end{array}$ & $\begin{array}{l}\text { - Higher rate of radiological changes in skiers } \\
\text { than controls (more degenerative disc changes } \\
\text { in alpine skiers) } \\
\text { - No significant difference in lifetime prevalence of } \\
\text { back pain between athletes and non-athletes } \\
\text { - The occurrence of MRI abnormalities in skiers is } \\
\text { not associated with lifetime prevalence of back } \\
\text { pain }\end{array}$ \\
\hline $\begin{array}{l}\text { Angoules et al., } \\
2018\end{array}$ & $\begin{array}{l}\text { - } 46 \text { non-professional } \\
\text { Greek classic } \\
\text { female ballet } \\
\text { dancers }\end{array}$ & Age: $28.8 \pm 5.44$ years & $\begin{array}{l}\text { Training hours/week: } \\
10.8 \pm 6.68 \\
\text { Years of experience: } \\
11.9 \pm 4.20\end{array}$ & & $\begin{array}{l}\text { - A self-administered } \\
\text { questionnaire }\end{array}$ & $\begin{array}{l}\text { - } 31 \text { dancers experienced } 3.26 \pm 1.7 \text { episodes of } \\
\text { mechanical LBP in the previous } 12 \text { months and } \\
\text { have to refrain from dancing activities } \\
\text { (16.9 } \pm 16.22 \text { days) } \\
\text { - } 21 \text { dancers received some kind of conservative } \\
\text { treatment }\end{array}$ \\
\hline
\end{tabular}




\begin{tabular}{|c|c|c|c|c|c|c|}
\hline \multicolumn{7}{|l|}{ TABLE 1 | Continued } \\
\hline Authors & $\begin{array}{l}\text { Sport and } \\
\text { performance } \\
\text { level }\end{array}$ & Subjects & Training & Back pain and/or injury & $\begin{array}{l}\text { Diagnostics of } \\
\text { back pain and/or } \\
\text { injury }\end{array}$ & Main findings \\
\hline Kraft et al., 2009 & $\begin{array}{l}\text { - } 58 \text { elite horseback } \\
\text { riders (18 men, } 40 \\
\text { women) } \\
\text { - Horseback riders } \\
\text { competed at a } \\
\text { national level, an } \\
\text { international level, } \\
\text { Olympic Games } \\
\text { and/or the World } \\
\text { Equestrian Games } \\
\text { - Control group: } 30 \\
\text { non-riding } \\
\text { volunteers (17 men, } \\
13 \text { women) }\end{array}$ & $\begin{array}{l}\text { Age of elite riders: } \\
32.4 \text { years } \\
\text { Age of controls: } \\
28.7 \text { years }\end{array}$ & $\begin{array}{l}\text { Training hours/week: } \\
18.5 \pm 8.2 \\
\text { Riding experience: } \\
20.1 \pm 9.1 \text { years }\end{array}$ & & $\begin{array}{l}\text { - Questionnaire } \\
\text { - Physical } \\
\text { examination } \\
\text { - MRI examination } \\
\text { - Visual Analogue } \\
\text { Scale }\end{array}$ & $\begin{array}{l}\text { - } 51 \text { elite horseback riders have a history of LBP } \\
\text { - The prevalence of LBP is not different among } \\
\text { disciplines } \\
\text { - The development of lumbar disk degeneration } \\
\text { is not associated with LBP history, discipline, } \\
\text { body mass index, and trunk/leg-length ratio } \\
\text { - No conclusive MRI evidence to suggest that the } \\
\text { cause of LBP in horseback riders lies in } \\
\text { structural changes of the spine }\end{array}$ \\
\hline $\begin{array}{l}\text { Adiele and Morgan, } \\
2018\end{array}$ & $\begin{array}{l}\text { - } 45 \text { competitive } \\
\text { swimmers ( } 22 \text { boys } \\
\text { and } 23 \text { girls) from } \\
\text { Zimbabwe }\end{array}$ & $\begin{array}{l}\text { Age: } \\
16.43 \pm 2.36 \text { years } \\
\text { Height: } 168.3 \pm 9.8 \mathrm{~cm} \\
\text { Weight: } \\
55.01 \pm 6.14 \mathrm{~kg}\end{array}$ & $\begin{array}{l}\text { Years of experience: } \\
3.41 \pm 1.52 \\
\text { Training duration: } \\
3.08 \pm 1.69 \mathrm{~h}\end{array}$ & & $\begin{array}{l}\text { - Epidemiological } \\
\text { survey }\end{array}$ & $\begin{array}{l}\text { - Musculoskeletal problems and pain in } 27 \\
\text { swimmers (16 male and } 11 \text { females) } \\
\text { - LBP in } 17.1 \% \text { males and } 26.2 \% \text { females }\end{array}$ \\
\hline $\begin{array}{l}\text { Koyama et al., } \\
2013\end{array}$ & $\begin{array}{l}\text { - } 104 \text { Japanese } \\
\text { collegiate gymnasts } \\
\text { (70 male and } 34 \\
\text { female) } \\
\text { - Pre-elite level } \\
\text { gymnasts (17) } \\
\text { - Elite level gymnasts } \\
\text { (71) } \\
\text { - National level } \\
\text { gymnasts (16). } \\
\text { - } 2 \text { gymnasts - } \\
\text { winners of medals } \\
\text { at Olympic and } \\
\text { World } \\
\text { Championships (4 } \\
\text { gold medals) }\end{array}$ & Age: $19.7 \pm 1.0$ years & $\begin{array}{l}\text { Training experience: } \\
11.8 \pm 3.6 \text { years } \\
\text { Approximately } 4 \mathrm{~h} \text { of } \\
\text { gymnastics training for } \\
6 \text { days/week }\end{array}$ & & $\begin{array}{l}\text { - Questionnaire } \\
\text { - MRI examination }\end{array}$ & $\begin{array}{l}\text { - More than } 1 \mathrm{MRI} \text { finding in } 49 \text { out of } 104 \\
\text { gymnasts } \\
\text { - High frequency of lumbar disc degeneration } \\
\text { (LDDG) and limbus vertebra is in gymnasts with } \\
\text { LBP compared to those without LBP } \\
\text { - Only LDDG is a significant variable predictor } \\
\text { associated with LBP }\end{array}$ \\
\hline
\end{tabular}


TABLE 2 | Training characteristics and identified back problems in athletes of team sports.

$\begin{array}{llll}\text { Farahbakhsh } & \text { - A cross-sectional } & \text { - Young top Iran } & \text { Age15.48 } \pm 1.81 \text { (other } \\ \text { et al., 2018 } & \text { survey } & \text { athletes; 452 male } & \text { sports) to } 16.1 \pm 0.93 \\ & \text { - Interview and } & \text { athletes participating } & \text { (soccer); } \\ & \text { questionnaire with 40 } & \text { in the sport Olympiad } & \text { Body height from } \\ & \text { questions related to } & \text { 2017 in Tehran } & 165.02 \pm 16.55 \text { (other } \\ \text { neck pain (NP) and to } & \text { Province } & \text { sports) to } 176.71 \pm 9.09 \\ & \text { LBP } & \text { Soccer }(n=136), & \text { (basketball); body weight } \\ & \text { volleyball }(n=81), & \text { from } 57.16 \pm 18.10 \text { (other } \\ & \text { basketball }(n=52), & \text { sports) to } 72.94 \pm 15.44 \\ & \text { wrestling }(n=69), & \text { (wrestling) } \\ & \text { other athletes }(n=39) & \end{array}$

other athletes $(n=39)$ $\begin{array}{ll}\text { Study design / } & \text { Sport and } \\ \text { diagnostics of back } & \text { performance level }\end{array}$

pain and/or injury

Fett et al., 2019 - A cross-sectional

survey

- Questionnaire
Training

Subjects

Age of athletes/controls

$19.7 \pm 4.7 / 21.2 \pm 2$ years

body height $181.9 \pm 12$

$180.1 \pm 8,9 \mathrm{~cm}$, body

weight

$74.5 \pm 14.8 / 74.0 \pm 10.3 \mathrm{~kg}$

Men/women controls

75:25\%/athletes 54:46\%

controls - sports

students $(n=166)$

Training volume

athletes/controls

$17.3 \pm 6.6 / 10.8 \pm 5 \mathrm{~h} /$ week

Number of competitions

$35.3 \pm 16.9 / 0$

experience $11.6 \pm 4.5$ years $/ 0$
Back pain and/or injury

Lifetime, 12-, 3-month, and point

prevalence of BP in athletes $85,75,58$,

$38 \%$, in controls $81,70,59 \%$

(non-significant differences)

- Non-significant differences between the sports games and controls

sports games

- BP location mostly identical for athletes and for controls, and in different sports games

- LB the most touched area, followed by the NP and upper back pain

- Correlations between BP and training load volume in all groups and pain location

Mean training hours/week $9.71 \pm 5.68$ (soccer),

$1.69 \pm 6.94$ (volleyball),

$11.29 \pm 4.64$ (wrestling),

$11.57 \pm 8.22$ (basketball),

$11.37 \pm 18.85$ (other sports)

Mean experience in sport

$5.1 \pm 2.53$ year (soccer)

$2.69 \pm 1.48$ years (volleyball),

$3.51 \pm 2.91$ years (wrestling),

$3.77 \pm 1.62$ years

(basketball),

$4.43 \pm 2.67$ years (other

sports
- Basketball players the highest life-time prevalence of NP (57.69\%)

- Volleyball players the highest life-time prevalence of LBP (67.90\%)

-Wrestlers the lowest life-time

prevalence of NP and LBP (18.84,

$$
\text { 10.14\%) }
$$

Basketball players the highest risk of $\mathrm{NP}$ in all time points (1.54-7.25)

- Basketball players the highest risk of LBP at most time points

-Wrestlers the lowest life-time risk of LBP (0.064-0.36)
Main findings

- The relationship between

raining volume and $\mathrm{BP}$

confirmed across all groups

.

.


TABLE 2 | Continued

\begin{tabular}{|c|c|c|c|c|c|c|}
\hline Authors & $\begin{array}{l}\text { Study design / } \\
\text { diagnostics of back } \\
\text { pain and/or injury }\end{array}$ & $\begin{array}{l}\text { Sport and } \\
\text { performance level }\end{array}$ & Subjects & Training & Back pain and/or injury & Main findings \\
\hline $\begin{array}{l}\text { Leppänen } \\
\text { et al., } 2015\end{array}$ & $\begin{array}{l}\text { - Retrospective analysis } \\
\text { as part of 3-year } \\
\text { follow-up study; } \\
\text { 12-month period } \\
\text { - A detailed } \\
\text { questionnaire, based } \\
\text { on a previous study of } \\
\text { sports injuries }\end{array}$ & $\begin{array}{l}-18 \text { of } 20 \text { basketball } \\
\text { and floorball teams of } \\
\text { the Tampere region; } \\
207 \text { basketball } \\
\text { players (boys } \\
n=101 \text {, girls } \\
n=106) ; 194 \text { floorball } \\
\text { players (boys } \\
n=112 \text {, girls } n=82 \text { ) }\end{array}$ & $\begin{array}{l}\text { - Floorball players/ basketball } \\
\text { players: age } 16.6 \pm 1.4 / \\
14.8 \pm 1.5 \text { years, BMl } \\
(21.9 \pm 2.2 / \\
21.4 \pm 3.0 \mathrm{~kg} / \mathrm{m}^{2}, \text { playing } \\
\text { experience } \\
7.6 \pm 3.0 / 6.9 \pm 2.9 \text { years }\end{array}$ & $\begin{array}{l}\text { Playing experience of } \\
\text { basketball players: boys/girls } \\
8.1 \pm 3.1 / 6.3 \pm 2.6 \text { years, } \\
\text { floorball players } 7.6 \pm 3.0 \text { vs. } \\
6.9 \pm 2.9 \text { years. } \\
\text { Training session/week of } \\
\text { boys/girls } \\
4.3 \pm 1.5 / 3.8 \pm 1.2 \text {; training } \\
\text { session/hour } \\
10.2 \pm 3.9 / 9.0 \pm 3.0 \text {; } \\
\text { game/season } \\
35.2 \pm 15.3 / 37.1 \pm 16.5\end{array}$ & $\begin{array}{l}\text { - Basketball: boys } 44 \text { ( } 45 \% \text { ) overuse } \\
\text { injuries, girls } 53 \text { ( } 55 \%) \text {, in total } 97,0.47 \\
\text { overuse injuries per athlete/year; most } \\
\text { of the overuse injuries at the lower } \\
\text { extremities ( } 64 \text { cases, } 66 \%), \text { knee ( } 44 \\
\text { cases, } 45 \%), \text { LBP ( } 27 \text { cases, } 13 \%) \\
\text { without differences between boys and } \\
\text { girls; average time loss from training } \\
\text { participation } 26 \pm 50 \text {, median } 7 \text { days } \\
\text { - Floorball: } 72 \text { ( } 37 \% \text { ) of players at least } \\
\text { one overuse injury ( } 51 \text { boys and } 21 \\
\text { girls); the most common injuries } \\
\text { location the lower back/pelvis ( } 36, \\
39 \%), \text { the second the knee (32, } 34 \%) ; \\
\text { boys significantly LB and knee overuse } \\
\text { injuries than girls; average time loss } \\
16 \pm 37 \text { (median 5) days; the ratio of } \\
\text { overuse injuries per athlete/year } 0.47\end{array}$ & $\begin{array}{l}\text { - The prevalence of overuse } \\
\text { injuries is rather high already in } \\
\text { youth basketball and floorball } \\
\text { categories }\end{array}$ \\
\hline $\begin{array}{l}\text { Anza et al., } \\
2013\end{array}$ & $\begin{array}{l}\text { - } 4 \text { months prospective } \\
\text { study } \\
\text { - Nordic musculoskeletal } \\
\text { questionnaire (NMQ) on } \\
\text { prevalence of } \\
\text { musculoskeletal } \\
\text { symptoms }\end{array}$ & Volleyball, sport clubs & $\begin{array}{l}\text { Boys }(n=19) \\
(185.6 \pm 6.72 \mathrm{~cm}) \\
\text { Girls }(n=19) \\
(171.8 \pm 9.28 \mathrm{~cm}) \\
14-17 \text { years. } \\
\text { Inclusion criterium: at least } \\
1 \text { year of regular training }\end{array}$ & $\begin{array}{l}\text { Weekly training hours: } \\
\text { boys } 10.78 \pm 1.76 \mathrm{~h} \text {, } \\
\text { girls } 14.11 \pm 2.08 \mathrm{~h}\end{array}$ & $\begin{array}{l}\text { - The most symptoms in } 4 \text { months found } \\
\text { in the back (56.5\%), shoulder (52.2\%), } \\
\text { hips/thighs ( } 52.2 \%) \text {, and knees (52.2\%) } \\
\text { - Small changes of the prevalence } \\
\text { regarding musculoskeletal symptoms } \\
\text { and pain in boys/girls in } 4 \text { months (LBP } \\
\text { in the last } 7 \text { days } 44.4 / 43 \% \text {, in the last } \\
12 \text { months } 55.0 / 56.5 \text {; players impaired } \\
\text { of sport activities } 17.4 / 11.1 \% \text {, } \\
\text { professional treatment of LBP } \\
11.1 / 13 \% \text { of players) }\end{array}$ & $\begin{array}{l}\text { - Training load was not a factor of } \\
\text { musculoskeletal symptoms and } \\
\text { pain }\end{array}$ \\
\hline $\begin{array}{l}\text { Augustsson } \\
\text { et al., } 2006\end{array}$ & $\begin{array}{l}\text { - Retrospective study } \\
\text { - Questionnaire designed } \\
\text { by the first author }\end{array}$ & $\begin{array}{l}\text { Volleyball, elite } \\
\text { Swedish division } \\
\text { players }\end{array}$ & $\begin{array}{l}\text { - } 225 \text { volleyball players of } 10 \\
\text { men's and } 9 \text { women's } \\
\text { teams } \\
\text { - Inclusion criterium: players } \\
\text { of regular team line-up } \\
\text { including substitutes } \\
\text { - Age: men } 25 \pm 4 \text { years; } \\
\text { women } 24 \pm 4 \text { years } \\
-47 \% \text { of men and } 53 \% \text { of } \\
\text { women returned the } \\
\text { questionnaire }\end{array}$ & $\begin{array}{l}\text { - The total time of training and } \\
\text { matches } 31,972 \mathrm{~h} \\
\text { - The number of training hours } \\
\text { per week for men } 9.9 \pm 4 \mathrm{~h} \\
\text { and } 9.4 \pm 7 \mathrm{~h} \text { for women } \\
\text { - Match playing time } 7 \mathrm{~h} / \text { week } \\
\text { for men and } 0.6 \mathrm{~h} / \text { week for } \\
\text { women }\end{array}$ & $\begin{array}{l}\text { - Most of the injuries in the ankle (23\%), } \\
\text { followed by knee (18\%) and back (15\%) } \\
\text { - Most injuries (62\%) of minor severity } \\
\text { - Most injuries during training (47\%), } 41 \% \\
\text { a gradual onset } \\
\text { - } 96 \% \text { of players performed prevention } \\
\text { training, mostly without supervision } \\
\text { (58\%) }\end{array}$ & $\begin{array}{l}\text { - Without distinguishing between } \\
\text { acute and overuse injuries, } \\
45 \% \text { of them occurred during } \\
\text { blocking, } 30 \% \text { during spiking }\end{array}$ \\
\hline
\end{tabular}




\begin{tabular}{|c|c|c|c|c|c|c|}
\hline Authors & $\begin{array}{l}\text { Study design / } \\
\text { diagnostics of back } \\
\text { pain and/or injury }\end{array}$ & $\begin{array}{l}\text { Sport and } \\
\text { performance level }\end{array}$ & Subjects & Training & Back pain and/or injury & Main findings \\
\hline $\begin{array}{l}\text { Bere et al., } \\
2015\end{array}$ & $\begin{array}{l}\text { - Prospective registration } \\
\text { of injuries during all } \\
\text { major FIVB } \\
\text { tournaments } \\
\text { - Medical reports }\end{array}$ & $\begin{array}{l}\text { - Junior and senior, } \\
\text { male and female } \\
\text { volleyball players at } \\
\text { major FIVB events } \\
\text { (23 senior and } 9 \\
\text { junior events) } \\
\text { - 4-year data obtained } \\
\text { through the FIVB } \\
\text { Injury Surveillance } \\
\text { System (ISS) }\end{array}$ & $\begin{array}{l}\text { - } 32 \text { events, } 2640 \text { of } 2710 \\
\text { report forms from team } \\
\text { medical staff, (response } \\
\text { rate of } 97.4 \%) \\
-440 \text { injuries reported } \\
\text { (62.5\% during match play, } \\
37.5 \% \text { during training) }\end{array}$ & & $\begin{array}{l}\text { - In all age and sex groups, the ankle the } \\
\text { most injured body part }(25.9 \%) \text {, } \\
\text { followed by knee (15.2\%), finger/thumb } \\
\text { (10.7\%), and lumbar/lower back ( } 8.9 \%) ; \\
\text { similar distribution between matches } \\
\text { and training } \\
\text { - The most common injury: joint sprains } \\
\text { (32.5\%, } n=143 \text { ) and muscle strains } \\
(14.1 \%, n=62) ; \text { most muscle strains in } \\
\text { the lower back ( } n=19) \\
\text { - Senior players a higher risk of injury } \\
\text { than juniors } \\
\text { - No difference between males and } \\
\text { females } \\
\text { - } 23.0 \% \text { of all injuries contact injuries, } \\
20.7 \% \text { overuse injuries, } 17.3 \% \\
\text { non-contact injury }\end{array}$ & $\begin{array}{l}\text { - Most injuries related to } \\
\text { sport-specific movement } \\
\text { patterns (repetitive jumping and } \\
\text { landing, spiking, blocking and } \\
\text { serving) }\end{array}$ \\
\hline $\begin{array}{l}\text { Bahr and } \\
\text { Reeser, } 2003\end{array}$ & $\begin{array}{l}\text { - Cohort study } \\
\text { - Retrospective injury } \\
\text { reports with } \\
\text { prospective registration } \\
\text { of injuries over a } \\
7.5 \text {-weeks } \\
\text { - Interview/report of } \\
\text { tournament medical } \\
\text { staff }\end{array}$ & $\begin{array}{l}\text { Beach volleyball, } \\
\text { players at the FIVB } \\
\text { World } \\
\text { Championships } 2001\end{array}$ & $\begin{array}{l}\text { Professional male and } \\
\text { female top beach volleyball } \\
\text { players ( } 178 \text { out of } 188 \\
\text { participants) from } 30 \\
\text { different countries }\end{array}$ & $\begin{array}{l}\text { - } 7.5 \text { weeks of training and } \\
\text { competitions before World } \\
\text { Championships ( } 6 \text { female } \\
\text { tournaments and } 8 \text { male } \\
\text { tournaments on the World } \\
\text { Tour, and other national or } \\
\text { regional tournaments) } \\
\text { - Men: } 2410 \text { match hours, } \\
1071 \text { warm up and } 6295 \text { h of } \\
\text { total training } \\
\text { - Women: } 1832 \text { match hours, } \\
814 \text { warm up and } 6493 \text { total } \\
\text { training hours }\end{array}$ & $\begin{array}{l}\text { - } 54 \text { of acute injuries recorded in the } \\
\text { retrospective survey, and } 25 \text { in the } \\
\text { prospective study } \\
\text { - } 67 \text { players ( } 38 \%) 79 \text { overuse injuries in } \\
\text { the retrospective survey with medical } \\
\text { attention } \\
\text { - Most common overuse injuries LBP } \\
\text { (15\%), knee pain (12\%), and shoulder } \\
\text { problems (10\%) } \\
\text { - Injuries in relation to body region in the } \\
\text { prospective study similar to that of } \\
\text { retrospective study anyway much lower }\end{array}$ & $\begin{array}{l}\text { - Low back (LB) and shoulder } \\
\text { overuse problems attributed to } \\
\text { frequent spiking and jump } \\
\text { serving } \\
\text { - LB problems in players depend } \\
\text { on the sand quality and its } \\
\text { depth, heavy wet balls when } \\
\text { raining (larger loads for the } \\
\text { shoulder and lumbar spine } \\
\text { during spiking and serving) }\end{array}$ \\
\hline $\begin{array}{l}\text { Külling et al., } \\
2014\end{array}$ & $\begin{array}{l}\text { - Cross sectional study } \\
\text { - Structured interview; } \\
\text { MRI examination }\end{array}$ & $\begin{array}{l}\text { - Beach volleyball; } \\
\text { - Grand Slam } \\
\text { Tournaments } \\
\text { - Fully professional } \\
\text { male players with or } \\
\text { without back pain; a } \\
\text { world ranking in the } \\
\text { top } 100\end{array}$ & $\begin{array}{l}\text { Age of players }(n=29) \\
28 \text { years }(19-39 \text { years), } \\
\text { body height } 187 \mathrm{~cm} \\
(179-205 \mathrm{~cm}), \text { body weight } \\
85 \mathrm{~kg}(63-100 \mathrm{~kg}), \text { BMl } \\
22.6 \mathrm{~kg} / \mathrm{m}^{2} \\
\left(19.7-25.6 \mathrm{~kg} / \mathrm{m}^{2}\right)\end{array}$ & $\begin{array}{l}\text { - The average duration of } \\
\text { professional beach volleyball } \\
\text { activity } 9.8 \text { years ( } 2-20 \text { years) } \\
\text { - The average training } \\
\text { hours/week } 18.5 \text { ( } 9-35 \text { h) }\end{array}$ & $\begin{array}{l}\text { - } 86 \% \text { of players LBP during their career, } \\
\text { in the last } 4 \text { weeks } 35 \% \\
\text { - Averaged pain at a visual analog scale } \\
\text { (VAS) } 3 \text { points (0-8) } \\
\text { - } 23 \text { out of } 29 \text { players ( } 79 \%) \text { at least } 1 \\
\text { degenerated disc of Pfirrmann grad >3; } \\
\text { the most affected spinal levels } L 4-5 \text { in } \\
14(48 \%) \text { and L5-S1 in } 15 \text { players } \\
(52 \%) \text { both levels involved in } 5 \text { players } \\
(17 \%) \\
-6(21 \%) \text { players a spondylolysis grade } 4 \\
\text { according to the Hollenburg } \\
\text { classification; spondylolisthesis in } 2 \\
\text { players } \\
\text { - Non-significant correlation between } \\
\text { LBP and MRI abnormalities }\end{array}$ & $\begin{array}{l}\text { - High prevalence of disc } \\
\text { degeneration and spondylolysis } \\
\text { in the lumbar spine in } \\
\text { professional beach volleyball } \\
\text { players } \\
\text { - No correlations between LBP } \\
\text { and MRI abnormalities in the } \\
\text { study subjects }\end{array}$ \\
\hline
\end{tabular}

urnaments on the World total training

iners $(n=29)$

fully profession

male players with or

without back pain;

orld ranking in the

kg (63-100 kg). BM

$2.6 \mathrm{~kg} / \mathrm{m}^{2}$ 


\begin{tabular}{|c|c|c|c|c|c|c|}
\hline Authors & $\begin{array}{l}\text { Study design / } \\
\text { diagnostics of back } \\
\text { pain and/or injury }\end{array}$ & $\begin{array}{l}\text { Sport and } \\
\text { performance level }\end{array}$ & Subjects & Training & Back pain and/or injury & Main findings \\
\hline $\begin{array}{l}\text { Bacon and } \\
\text { Mauger, } 2017\end{array}$ & $\begin{array}{l}\text { - Prospective cohort } \\
\text { study } \\
\text { - Volume and intensity } \\
\text { variables derived from } \\
\text { GPS (StatSports, Viper } \\
\text { Pod, NI) } \\
\text { - Injuries collected by a } \\
\text { qualified } \\
\text { physiotherapist }\end{array}$ & $\begin{array}{l}\text { - Professional youth } \\
\text { soccer players } \\
(n=41), \text { Barclays } \\
\text { U21/U18 Premier } \\
\text { Leagues } \\
\text { - Data collection } \\
40 \text { weeks of the } \\
\text { competitive season } \\
\text { for training sessions } \\
\text { and matches }\end{array}$ & $\begin{array}{l}\text { - Season } 2012 / 2013 \\
(n=18) \text {, age } \\
18.7 \pm 1.2 \text { years, body } \\
\text { height } 175.2 \pm 4.5 \mathrm{~cm}, \\
\text { body weight } 72.4 \pm 3.1 \mathrm{~kg} \\
\text { - Season } 2013 / 2014 \\
(n=23) \text {, age } \\
17.0 \pm 1.1 \text { years, body } \\
\text { height } 181.3 \pm 6.1 \mathrm{~cm}, \\
\text { body weight } 74.9 \pm 8.7 \mathrm{~kg}\end{array}$ & $\begin{array}{l}\text { - Players either with a signed } \\
\text { youth scholarship contract or } \\
\text { a professional contract with } \\
\text { the club } \\
\text { - } 6 \text { training sessions per week }\end{array}$ & $\begin{array}{l}\text { - Of the } 190 \text { injuries } 5.46 \text { IIR, } 51.76 \% \\
(n=44,5) \text { non-contact } \\
\text { - Total distance significantly predict } \\
\text { overuse injury rates }\left(F_{1,39}=6.482\right. \\
p=0.015), \text { high-speed running meters } \\
\text { not }\left(F_{1,39}=1.003, p=0.323\right) \\
\text { - Back injuries very low rate } \\
(0.25 / 1000 \mathrm{~h}, 2.4 \%)\end{array}$ & $\begin{array}{l}\text { - The incidence of overuse injury } \\
\text { impact distance covered in } \\
\text { training and matches, not the } \\
\text { high-speed running meters } \\
\text { - Cumulative approach to training } \\
\text { load has value when assessing } \\
\text { players injury risk }\end{array}$ \\
\hline $\begin{array}{l}\text { Pasanen et al., } \\
2008\end{array}$ & $\begin{array}{l}\text { - } 6 \text { months prospective } \\
\text { study } \\
\text { - Structured } \\
\text { questionnaire } \\
\text { registered by player } \\
\text { and verified personally } \\
\text { by a physician }\end{array}$ & $\begin{array}{l}\text { Floorball, licensed } \\
\text { female players from } \\
\text { Finnish top leagues } \\
(n=374) \\
\text { - Players' level: elite } \\
\text { league }(n=164), \text { first } \\
\text { division }(n=183), \\
\text { second division } \\
(n=27), \\
\text { - Field position: } \\
\text { goalkeeper } n=41 \\
\text { defender } n=120, \\
\text { forward } n=213\end{array}$ & $\begin{array}{l}\text { Age } 24.2 \pm 4.8 \text { years, body } \\
\text { height } 166.22 \pm 5.2 \mathrm{~cm} \text {, } \\
\text { body weight } 61.6 \\
2 \pm 6.5 \mathrm{~kg}\end{array}$ & $\begin{array}{l}\text { - } 122 \text { practice hours and } 5.9 \\
\text { match hours/player in } \\
\text { average } \\
\text { - Elite league } 133 \text { practice } \\
\text { hours }(95 \% \mathrm{Cl} \\
124.9-141.5 \mathrm{~h} \text { ), first division } \\
111 \mathrm{~h}(95 \% \mathrm{Cl} \\
\text { 103.9-117.9 h), second } \\
\text { division } 124 \mathrm{~h}(95 \% \mathrm{Cl} \\
\text { 103.9-143.7) } \\
\text { - Intensive game load average } \\
\text { during the season: elite } \\
\text { league players } 7.5 \mathrm{~h}(95 \% \mathrm{Cl} \\
\text { 6.8-8.1 h), first-division } \\
\text { players } 4.7(95 \% \mathrm{Cl} \\
\text { 4.2-5.1 h), second-division } \\
\text { players } 4.7 \mathrm{~h}(95 \% \mathrm{Cl} \\
\text { 3.8-5.6 h) }\end{array}$ & $\begin{array}{l}-73 \% \text { of the injuries traumatic and } 27 \% \\
\text { from overuse } \\
\text { - Most of the traumatic injuries in ankle } \\
\text { and knee }(29 / 28 \%) \\
\text { - Most of the overuse injuries in knee, } \\
\text { calf/shin ( } 22 \% \text { ) and back (14\%) } \\
\text { - The injury rate per practice hours } \\
1.8 / 1000 \\
\text { - Overall injury rate/1000 game hours } \\
40.3 \text {, in the elite league } 34.3 \text {, in the first } \\
\text { division } 48.0 \text { and in the second division } \\
47.7 \\
-56 \% \text { of injuries treated at clinics, } 44 \% \\
\text { by the players themselves, } 6 \% \text { needed } \\
\text { hospital admission }\end{array}$ & $\begin{array}{l}\text { - No clear reason of a high } \\
\text { incidence of overuse injuries } \\
\text { and non-contact injuries } \\
\text { - Possible factors - type of floor } \\
\text { and shoes, lack of } \\
\text { proprioception and conditioning } \\
\text { training }\end{array}$ \\
\hline $\begin{array}{l}\text { Haydt et al., } \\
2012\end{array}$ & $\begin{array}{l}\text { - Cross sectional study } \\
\text { - Pen and paper survey } \\
\text { questionnaire on } \\
\text { incidence of LBP }\end{array}$ & $\begin{array}{l}\text { - Female NCAA } \\
\text { Division III field } \\
\text { hockey players (FH); } \\
\text { season } 2008(n=90) \text {; } \\
\text { age-matched } \\
\text { controls from } \\
\text { Misericordia } \\
\text { University ( } n=98) \text {, } \\
\text { without participation } \\
\text { in FH } \\
\text { - Inclusion criteria: } \\
\text { females 18-24 years } \\
\text { with a self-reported } \\
\text { LBP lasting more } \\
\text { than } 24 \text { h not } \\
\text { associated with } \\
\text { menstruation }\end{array}$ & $\begin{array}{l}\text { Age } \\
\text { FH players } \\
19.28 \pm 1.19 \text { years; range } \\
18-22 \text { years; controls } \\
19.89 \pm 1.45 \text { years; range } \\
18-24 \text { years }\end{array}$ & $\begin{array}{l}\text { - Not mentioned although they } \\
\text { were surveyed }\end{array}$ & $\begin{array}{l}\text { - Similarity in incidence of LBP in the FL } \\
\text { players and controls ( } 56 \% \text { in FL } \\
\text { players, } 55 \% \text { in controls) } \\
\text { - Onset age of LBP for controls and the } \\
\text { FH was } 16.45 \pm 2.12 \text { and } \\
16.23 \pm 1.80 \text { years } \\
\text { - Duration of symptoms was less than } \\
3 \text { weeks in controls ( } 85 \% \text { ) and FH } \\
\text { group ( } 82 \%) \\
\text { - Episode of LBP of } 1 \text { month or longer } \\
\text { was } 15 \% \text { in controls and } 14 \% \text { in FH } \\
\text { - Similarities in pain distal to the buttock } \\
\text { in both groups }\end{array}$ & $\begin{array}{l}\text { - Non-significant differences in } \\
\text { the incidence of LBP and pain } \\
\text { characteristics between the } \\
\text { groups } \\
\text { - In both groups a high incidence } \\
\text { of LBP (>50\%) with an onset of } \\
\text { LBP at a mean age of } \\
\text { approximately } 16 \text { years }\end{array}$ \\
\hline
\end{tabular}


TABLE 2 | Continued

\begin{tabular}{|c|c|c|c|c|c|c|}
\hline Authors & $\begin{array}{l}\text { Study design / } \\
\text { diagnostics of back } \\
\text { pain and/or injury }\end{array}$ & $\begin{array}{l}\text { Sport and } \\
\text { performance level }\end{array}$ & Subjects & Training & Back pain and/or injury & Main findings \\
\hline $\begin{array}{l}\text { Jacobson and } \\
\text { Tegner, } 2007\end{array}$ & $\begin{array}{l}\text { - Prospective cohort } \\
\text { study } \\
\text { - Injuries registered by } \\
\text { trainer/coach, in } \\
\text { national teams by } \\
\text { physiotherapist using } \\
\text { standardized protocols } \\
\text { (Ekstrand, 1982) } \\
\text { - Injured players } \\
\text { interviewed by the first } \\
\text { author by telephone }\end{array}$ & $\begin{array}{l}\text { Swedish female elite } \\
\text { soccer players in } \\
\text { premiere league, } \\
\text { divided in national } \\
(n=51) \text { and } \\
\text { no-national players } \\
(n=144)\end{array}$ & $\begin{array}{l}\text { - } 195 \text { of } 269 \text { players }(72 \%) \\
\text { - Age } 23 \pm 4 \text { years, body } \\
\text { height } 168 \pm 5 \mathrm{~cm} \text {, body } \\
\text { weight } 62 \pm 7 \mathrm{~kg}, \mathrm{BMl} \\
22 \pm 2\end{array}$ & $\begin{array}{l}\text { - On the average } 140 \pm 48 \\
\text { (24-238) practice sessions } \\
\text { and } 35 \pm 12(2-48) \text { matches } \\
\text { - Players of national teams - } \\
52 \text { practice sessions and } 23 \\
\text { matches more } \\
\text { - Total football hours } \\
\text { national/non-national players } \\
321 \pm 134 / 257 \pm 78 \mathrm{~h}\end{array}$ & $\begin{array}{l}\text { - Traumatic injuries } 163 / 237 \text { ( } 69 \% \text {, } \\
3.3 / 1000 \mathrm{~h} \text { of football) } \\
\text { - Overuse injuries } 74 / 237 \text { (1.3/1000 h of } \\
\text { soccer) } \\
\text { - Overuse injuries mostly during pre- and } \\
\text { at the beginning of spring season } \\
\text { - The highest incidence in the knee, the } \\
\text { lower leg, and the back (39/18/16\%; } \\
0.6,0.3 \text {, and } 0.2 / 1000 \text { h of soccer, } \\
\text { respectively }\end{array}$ & $\begin{array}{l}\text { - Back the third of most common } \\
\text { body location of acute and } \\
\text { overuse injuries } \\
\text { - National team players and } \\
\text { players in the three highest } \\
\text { ranked teams although } \\
\text { exposed to more playing hours } \\
\text { during the year not different in } \\
\text { injury incidence as non-national } \\
\text { players } \\
\text { - Injured players higher } \\
\text { practice/game ratio than } \\
\text { non-injured players } \\
\text { - High amount of re-injuries } \\
\text { (>50\%) }\end{array}$ \\
\hline $\begin{array}{l}\text { Tunås et al., } \\
2015\end{array}$ & $\begin{array}{l}\text { - Prospective cohort } \\
\text { study } \\
\text { - Standardized Nordic } \\
\text { musculoskeletal } \\
\text { questionnaire (NMQ) }\end{array}$ & $\begin{array}{l}\text { - Female elite soccer } \\
\text { players (2009-2011; } \\
3 \text { years) }(n=267) \\
\text { - Female elite handball } \\
\text { players (2007-2011; } \\
5 \text { years) }(n=173) \\
\text { - Female controls } \\
\text { (2012) }(n=400) \\
\text { practicing no more } \\
\text { than } 5 \text { times per } \\
\text { week }\end{array}$ & $\begin{array}{l}\text { Soccer/handball } \\
\text { players/controls: } \\
\text { Mean age } \\
22.4 \pm 4 / 22.3 \pm 3 / \\
25.6 \pm 4 \text { years, } \\
\text { Body height } \\
167.7 \pm 5 / 173.1 \pm 6 / \\
167.9 \pm 7 \mathrm{~cm}, \\
\text { Body weight } \\
62.6 \pm 7 / 69.0 \pm 7 / \\
66.1 \pm 14 \mathrm{~kg}, \\
\text { Playing at elite level } \\
3.1 \pm 4 / 3.2 \pm 4 \text { years }\end{array}$ & $\begin{array}{l}\text { Training volume of players: } \\
\text { - Soccer: less than } 400 \mathrm{~h} / \text { year } \\
5.2 \% \text {; } 400-699 \mathrm{~h} / \text { year } \\
\text { 74.5\%; more than } 700 \mathrm{~h} / \text { year } \\
\text { 20.3\% of players; } \\
\text { - Handball less than } 400 \mathrm{~h} / \text { year } \\
\text { 5.8\%; 400-699 h/year } \\
73.4 \% \text {; more than } 700 \mathrm{~h} / \text { year } \\
\text { 20.8\% of players }\end{array}$ & $\begin{array}{l}\text { - No differences in the prevalence of LBP } \\
\text { among the groups } \\
\text { - More than } 60 \% \text { players of the groups } \\
\text { experienced LBP ever, more than } 50 \% \\
\text { during previous } 12 \text { months and } \\
24-31 \% \text { in the previous week } \\
\text { - Only } 3.2 \% \text { of handball, } 3.6 \% \text { of football } \\
\text { players and } 4.9 \% \text { controls had no } \\
\text { experience with LBP } \\
\text { - More than } 70 \% \text { of football and handball } \\
\text { players with LBP stated as the source } \\
\text { the overuse, less than } 10 \% \text { traumatic } \\
\text { injury }\end{array}$ & $\begin{array}{l}\text { - Non-significant differences in } \\
\text { the prevalence of LBP among } \\
\text { soccer players, handball } \\
\text { players and the control group } \\
\text { - High frequency of LBP among } \\
\text { examined players; as the main } \\
\text { source of LBP stated overuse }\end{array}$ \\
\hline $\begin{array}{l}\text { Haag et al., } \\
2016\end{array}$ & $\begin{array}{l}\text { - Cross-sectional survey } \\
\text { based on a } \\
\text { retrospective data } \\
\text { - An iPad-compatible } \\
\text { questionnaire; a } \\
\text { multiple-logistic- } \\
\text { regression model } \\
\text { - Nomogram based on } \\
\text { the ORs of each } \\
\text { parameter }\end{array}$ & $\begin{array}{l}841 \text { male, } 269 \text { female } \\
\text { soccer players from } \\
\text { Bavarian soccer } \\
\text { clubs ( } 74 \text { different } \\
\text { clubs, } 75.0 \% \text { from } \\
1480 \text { athletes) }\end{array}$ & $\begin{array}{l}\text { Age of boys } \\
15.96 \pm 1.52 \text { years, girls } \\
14.38 \pm 1.67 \text { years, body } \\
\text { height of boys } \\
174.96 \pm 9.03 \mathrm{~cm} \text {, girls } \\
163.82 \pm 6.64 \mathrm{~cm} \text {; body } \\
\text { weight of boys } \\
64.59 \pm 11.74 \mathrm{~kg} ; \text { girls } \\
52.64 \pm 9.11 \mathrm{~kg}\end{array}$ & $\begin{array}{l}\text { Mean soccer training in boys } \\
8976 \pm 2.94 \text { years } \\
(7-11 \text { years) and in girls } \\
3986 \pm 3.5 \text { years ( } 3-8 \text { years) }\end{array}$ & $\begin{array}{l}\text { - The greatest impact on prevalence of } \\
\text { back pain (BP) the age, followed by sex } \\
\text { and playing surface (U19/U17/U15, } \\
\text { 1.84/1.66/1.11) } \\
\text { - Female players a greater risk } \\
\text { (OR }=1.48 \text { ) for BP than the boys; } \\
\text { higher BMl linked to lower amount of } \\
\text { BP (OR }=0.97) \text {; longer training history a } \\
\text { negative effect on BP (OR }=1.04) ; \\
\text { more than } 6 \text { or less than } 3 \text { training } \\
\text { h/week significant effects on the } \\
\text { prevalence of BP (OR }=0.81) \text {; natural } \\
\text { grass the highest negative influence } \\
\text { (OR }=1.56) ; \text { goalkeepers specific injury } \\
\text { prevalence and incidence }\end{array}$ & $\begin{array}{l}\text { Among all factors, longer } \\
\text { training history and very high or } \\
\text { low training load have an } \\
\text { impact on the prevalence of BP }\end{array}$ \\
\hline
\end{tabular}




\begin{tabular}{|c|c|c|c|c|c|c|}
\hline Authors & $\begin{array}{l}\text { Study design / } \\
\text { diagnostics of back } \\
\text { pain and/or injury }\end{array}$ & $\begin{array}{l}\text { Sport and } \\
\text { performance level }\end{array}$ & Subjects & Training & Back pain and/or injury & Main findings \\
\hline $\begin{array}{l}\text { Bowen et al., } \\
2017\end{array}$ & $\begin{array}{l}\text { - } 2 \text { year prospective } \\
\text { study } \\
\text { - Workload quantified } \\
\text { using GPS (Viper V.2, } \\
\text { StatSports, Ireland), } \\
\text { data from all on-pitch } \\
\text { training sessions and } \\
\text { matches } \\
\text { - Injury report classified } \\
\text { by doctors and } \\
\text { physiotherapists }\end{array}$ & $\begin{array}{l}\text { Elite youth soccer } \\
\text { players }(n=32) \\
\text { category academy } \\
\text { U18, U21 Premier } \\
\text { League 2013-2014 } \\
\text { and 2014-2015 } \\
\text { seasons }\end{array}$ & $\begin{array}{l}\text { Age } 17.3 \pm 0.9 \text { years, body } \\
\text { height } 180.0 \pm 7.3 \mathrm{~cm} \text {, } \\
\text { body weight: } 74.1 \pm 7.0 \\
\mathrm{~kg}\end{array}$ & $\begin{array}{l}\text { - Training on a full-time basis } \\
\text { - Competition: } 63 \% \text { of } \\
\text { participants in both seasons } \\
\text { and } 38 \% \text { participants in one } \\
\text { season }\end{array}$ & $\begin{array}{l}\text { - Very high number of accelerations } \\
(\geq 9254) \text { over } 3 \text { weeks associated with } \\
\text { the highest relative risk (RR) }(R R=3.84) \\
\text { and non-contact injury risk (RR }=5.11) \\
\text { - Non-contact injury risk higher by } \\
\text { combination of high acute high-speed } \\
\text { distance }(\mathrm{HSD}) \text { with low chronic HSD } \\
\text { (RR }=2.55), \text { but not with high chronic } \\
\text { HSD (RR }=0.47) \\
\text { - Most common non-contact injuries in } \\
\text { ankle/foot }(2.1 / 1000 \mathrm{~h}), \text { hip/groin } \\
(1.3 / 1000 \mathrm{~h}), \mathrm{knee}(1.1 / 1000 \mathrm{~h}) \text {, and } \\
\text { abdomen/back }(0.4 / 1000 \mathrm{~h})\end{array}$ & $\begin{array}{l}\text { - High accumulated and acute } \\
\text { workloads are associated with } \\
\text { a greater injury risk } \\
\text { - The injury risk is greatest by } \\
\text { very high number of } \\
\text { accelerations accumulated over } \\
3 \text { weeks } \\
\text { - Progressive increases of } \\
\text { chronic workload develops the } \\
\text { players' tolerance to higher } \\
\text { acute loads and reduces injury } \\
\text { risk }\end{array}$ \\
\hline $\begin{array}{l}\text { Aasheim et al., } \\
2018\end{array}$ & $\begin{array}{l}\text { - Prospective cohort } \\
\text { study (7 months) } \\
\text { - OSTRC-O } \\
\text { questionnaire } \\
\text { distributed by email } \\
\text { every second Sunday, } \\
\text { software SurveyXact } \\
\text { V.8.2, Rambø\|ll } \\
\text { Management } \\
\text { Consulting, Oslo, } \\
\text { Norway }\end{array}$ & $\begin{array}{l}\text { - Male handball players } \\
\text { aged 16-18 years } \\
(n=145) \text { from } \\
\text { South-Eastern } \\
\text { Norway during the } \\
\text { 2016-2017 season } \\
\text { - Complete data from } \\
\text { 48\% of players }\end{array}$ & $\begin{array}{l}\text { - } 61 \% \text { of players } 17 \text { years } \\
\text { old, } 33 \% 18 \text { years old and } \\
6 \% 16 \text { years old; body } \\
\text { height } 184 \pm 7 \mathrm{~cm} \\
(172-198 \mathrm{~cm}), \text { body weight } \\
80 \pm 11 \mathrm{~kg}(60-115 \mathrm{~kg}) \\
-55 \% \text { of players participated } \\
\text { in two different teams } \\
\text { (senior/junior), } 28 \% \text { in one } \\
\text { team, } 17 \% \text { in three teams, } \\
10 \% \text { players in junior } \\
\text { national team }\end{array}$ & $\begin{array}{l}\text { - Handball training: } 9 \pm 2 \text { years } \\
\text { (4-14 years). } \\
\text { - The median amount of } \\
\text { training during the study: } \\
14 \mathrm{~h} / \text { week, approximately } 6 \mathrm{~h} \\
\text { handball, } 4.5 \mathrm{~h} \text { strength, } 3 \mathrm{~h} \\
\text { alternative training and } \\
30 \text { min handball match play }\end{array}$ & $\begin{array}{l}\text { - Average prevalence of all overuse } \\
\text { injuries } 39 \% \text { ( } 95 \% \mathrm{Cl} 29-49) \\
\text { - Average prevalence of all substantial } \\
\text { overuse injury problems } 15 \% \text { ( } 95 \% \mathrm{Cl} \\
\text { 13-17) } \\
\text { - Highest average prevalence in shoulder } \\
\text { (38\% of players), knee (36\%), and low } \\
\text { back (26\%); overuse injuries in each } \\
\text { anatomical area after } 7 \text { months about } \\
20 \% \text { higher than at baseline }\end{array}$ & $\begin{array}{l}\text { - The prevalence of overuse } \\
\text { injuries is high, especially in the } \\
\text { shoulder, knee, and low back } \\
\text { - Tendency of worsening during } \\
\text { the season }\end{array}$ \\
\hline $\begin{array}{l}\text { Hoskins et al., } \\
2009\end{array}$ & $\begin{array}{l}\text { - A cross-sectional study } \\
\text { - The questionnaire using } \\
\text { Quadruple Visual } \\
\text { Analogue Scale of the } \\
\text { McGill Pain } \\
\text { Questionnaire } \\
\text { (short-form) with LBP } \\
\text { questions adapted from } \\
\text { an Australian LBP } \\
\text { epidemiological study }\end{array}$ & $\begin{array}{l}\text { - } 271 \text { elite players, } 360 \\
\text { semi-elite players, } \\
148 \text { non-athletic } \\
\text { controls } \\
\text { - At the elite/semi-elite } \\
\text { level Australian rugby } \\
\text { league players } \\
(n=93 / 52), \text { rugby } \\
\text { union players } \\
(n=19 / 139), \\
\text { Australian-Rules } \\
\text { players }(n=112 / 90) \\
\text { and soccer players } \\
(n=47 / 70)\end{array}$ & $\begin{array}{l}\text { Age of } \\
\text { elite/semi-elite/non-athletic } \\
\text { controls } \\
23.3 \pm 4 \text { years, } \\
23.8 \pm 4 \text { years, and } \\
23.9 \pm 4.5 \text { years }\end{array}$ & - & $\begin{array}{l}\text { - Almost linear trend of increasing LBP } \\
\text { severity from non-athletes, to the } \\
\text { semi-elite and elite athletes } \\
\text { - Levels of the sensory, affective and total } \\
\text { pain score significantly higher in elite } \\
\text { athletes } \\
\text { - Elite athletes approximately two times } \\
\text { more experienced discomforting or } \\
\text { greater LBP, and less likely no LBP as } \\
\text { other groups; semi-elite athletes less } \\
\text { likely experienced discomforting or } \\
\text { greater LBP; and non-athletes more } \\
\text { likely not experienced LBP ( } \chi^{2}=18.67 \text {, } \\
p<0.001) \\
\text { - The age of first episode of LBP - } \\
\text { between groups non-significant } \\
\text { differences } \\
\text { - Elite athletes } 2-4 \text { times attributed } \\
\text { sporting activity as a cause of current } \\
\text { LBP }\end{array}$ & $\begin{array}{l}\text { - Elite players had significantly } \\
\text { higher levels and more frequent } \\
\text { LBP } \\
\text { - LBP in elite players was more } \\
\text { attributed to sporting activity } \\
\text { when compared to semi-elites } \\
\text { and non-athletes } \\
\text { - The difference of onset of the } \\
\text { first time LBP non-significant } \\
\text { between groups } \\
\text { - Reported etiology between } \\
\text { groups is different }\end{array}$ \\
\hline
\end{tabular}


1999; Pasanen et al., 2008; Hoskins et al., 2009; Haydt et al., 2012; Külling et al., 2014; Fett et al., 2019). Among pain caused by overuse, low back pain was the most often reported pain with an incidence of $20-86 \%$ depending on the age category, sex, performance level, and the time of occurrence. In soccer, volleyball, and handball, low back pain was the third most common pain caused by overuse (Augustsson et al., 2006; Anza et al., 2013; Bere et al., 2015; Tunås et al., 2015; Bowen et al., 2017; Aasheim et al., 2018). In soccer, pain caused by overuse of groin and hip with a rate of $38-57 \%$ was the most common, followed by low back pain caused by overuse, whereas in volleyball and handball overuse injuries of shoulder and knee at a rate of 46$57 \%$ and $12-59 \%$, respectively, were the most common, followed by low back pain.

The risk factors also include training and match load, which is characterized by the specific movement pattern of sport games, such as unilateral load, repetitive static flexion, hyperextension combined with rotation of the spine, jumps, impacts etc. (Snellman et al., 2001; Pasanen et al., 2008; Bere et al., 2015; Fett et al., 2019). However, the evidence is not unambiguous. The findings are mostly based on comparisons of athletes and non-athletes, and players with other athletes, top, elite, and nonelite players (Bahr and Reeser, 2003; Jacobson and Tegner, 2007; Hoskins et al., 2009; Anza et al., 2013; Bere et al., 2015; Haag et al., 2016; Aasheim et al., 2018).

In recent years, more attention has been directed toward objective quantification of training and match load, especially using the ACWR, and the examination of its relationship with injury risk (Griffin et al., 2020). Although 15 studies dealt with this issue in team sport games, only 2 of them can be included in this review (Bacon and Mauger, 2017; Bowen et al., 2017). In other studies, there was no distinction between the type and site of injuries.

Overall, the literature supports the association of training load with the occurrence of back problems in athletes of individual and team sports. A review by Moradi et al. (2015) identified many potential general and sport-specific risk factors for back pain in athletes, however, the evidence exists only for previous low back pain, decreased lumbar extension or flexion, and high body weight. Differences in the association between potential risk factors and back pain may be ascribed to the type of sport, level of competition, and training characteristics (Moradi et al., 2015). Evidently, properly designed training programs might not affect postural and core stability or increase the risk of injuries. For instance, lower lumbar lordosis and thoracic kyphosis, as well as anterior pelvic tilt while standing were identified in Latin American style professional dancers (Muyor et al., 2017). Though specific postures and movements in dance modify their spinal curvatures, it does not alter the spinal morphology in standing when compared to non-dancers. Further, Belavý et al. (2017) demonstrated that chronic running is associated with better intervertebral discs (IVD) composition and IVD hypertrophy. Generally, pain perception and processing is different in athletes as compared to normally active controls (Tesarz et al., 2012). This suggests a compensatory response of the endogenous antinociceptive system to the noxious input during exhaustive endurance training (Tesarz et al., 2013). More specifically, an overstressing of the endogenous pain inhibitory pathways may lead to exhaustion over time (Tesarz et al., 2013). This may result in disinhibition of pain processing when the acute pain is transmitted in the chronic pain and spatial pain spreading (Tesarz et al., 2013). On the contrary, the endogenous pain inhibitory pathways may be protected from chronic overstressing over time by a shift in the activation threshold, which may increase the efficiency of pain inhibition (Tesarz et al., 2013). Specific changes in pain thresholds and higher tolernce to pain at rest in athletes (Tesarz et al., 2012) has to be also taken into account when interpreting self-reported back pain and its relationship with physiological loading of the spine in athletes.

Actually, training is a protective factor against injury. As shown, high chronic workloads decrease the injury risk (Gabbett and Domrow, 2005). A lower risk of sustaining a subsequent injury was reported in athletes of team sports when they trained over 18 weeks before their initial injury (Gabbett and Domrow, 2005). A decreased risk of injury is also associated with welldeveloped physical qualities (Quarrie et al., 2001; Gabbett and Domrow, 2005; Gabbett et al., 2012; Gastin et al., 2015). Thus, progressively increased high training loads not only improve physical fitness of players but may also protect against injury (Gabbett, 2016). For instance, heavier rugby players with faster speed generate greater impact forces which may increase the recurrent rates of contact injuries (Gabbett et al., 2012). However, greater intermittent running speed at high intensity as well as muscle strength and power may decrease the risk of injuries in these players (Gabbett et al., 2012). This indicates that not only overtraining but also undertraining may increase the injury risk (Lyman et al., 2001; Orchard et al., 2009; Cross et al., 2016).

So far, the relationship between the ACWR and back pain/or injury in athletes remains unclear. This is mainly due to the limited evidence and varying methodological quality of particular studies. Nonetheless, it is most likely that sudden spikes in acute training loads may have detrimental effects, whereas high chronic loads and adequate strength and endurance of core muscles could provide protective effects on increasing the occurrence of back pain and/or injury. A structured back-strengthening program has been recommended for reduction of back pain experienced by athletes (Trainor and Wiesel, 2002). In practice, however, functional strength training for back pain prevention can be similar to programs for back pain rehabilitation (Wirth et al., 2017). Most exercises have not been tested for their effectiveness and compared with the load used for strength training. Neither are core strength exercises more effective than traditional resistance exercises (Smith et al., 2014) in individuals with back pain (Macedo et al., 2016; Saragiotto et al., 2016). Adaptations in voluntary activation of trunk muscles to core stability exercises have provided a basis for exercise guidelines (Wirth et al., 2017). However, adaptations of morphological structures that are essential for the core stability have not been sufficiently addressed in research studies. Therefore, the guidelines used for rehabilitation of back pain are insufficient for strength training in athletes (Wirth et al., 2017). Currently, there is no evidence-based exercise program for athletes with back pain and/or injury. Most studies investigated only a part with a narrow group of athletes who usually performed different types of exercise (Daniels et al., 
2020). Recent evidence suggests that poor load management is a main injury risk factor (Soligard et al., 2016). Recently, the IOC provided the consensus statement that include guidelines for monitoring of training, competition and psychological load, prescription of training and competition load, athlete well-being and injury (Soligard et al., 2016).

\section{Gaps in Current Studies Investigating the Workload in Relation to Back Problems in Athletes and Proposals for Future Research}

One of the former studies reported that the incidence of injury is increased when the duration, intensity, and load of training sessions and matches in rugby league is increased (Gabbett, 2004a; Gabbett and Domrow, 2007). There is a U-shaped relationship for 4-week cumulative loads with an increase in injury risk with higher loads (Cross et al., 2016). The risk of injury in professional rugby union players increases if they have high 1-week cumulative loads or large week-to-week changes in the training load (Cross et al., 2016). Further, higher risk of injury is also associated with a large increase in acute workload in elite cricket fast bowlers (Hulin et al., 2014). A sharp increases in running workload, the ACWR $>2.0$ for either total distance or a high-speed distance, increases the likelihood of injury in elite footballers in both a given and the subsequent week (Murray et al., 2017). Controlling for training load in a given week may decrease the odds of injury in the subsequent week. Windt et al. (2017) demonstrated that increasing participation in preseason training may decrease the risk of in-season injury in elite rugby league players. Though increased amounts of high-velocity running are associated with higher risk of soft-tissue injury of the lower body, distances covered at moderate and low speeds are protective against this injury (Gabbett and Ullah, 2012). Decreasing the training load during an early-competition phase can reduce the odds of injury without compromising the agility performance in athletes of collision sports (Gabbett and Domrow, 2007). Likewise, reductions in pre-season training loads decrease training injury rates and at the same time increase the maximal aerobic power in rugby league players (Gabbett, 2004b). It seems that high chronic training loads may decrease the risk of injuries in athletes. For instance, elite rugby league players are more resistant to injury when train at a high than a low chronic workload with ACWRs $0.85-1.35$, whereas they are less resistant to injury when ACWRs is $\sim 1.5$ and they are subjected to "spikes" in acute workload (Hulin et al., 2016b). Very high and high chronic workloads have protective effects against match injury following shorter recovery periods between matches (Hulin et al., 2016a). Alternatively, a high aerobic capacity and playing experience protect injury in elite Gaelic football players against rapid changes in workload and high ACWRs > 2.0 (Malone et al., 2017a). Players better tolerated increased distances and exposures to maximal velocity when train at higher than at lower chronic loads (Malone et al., 2017b). Over- and under-exposure them to maximal velocity increased the injury risk (Malone et al., 2017b). Accordingly, high chronic workloads, combined with reductions in acute workloads before competition, decrease the risk of injury but might also improve sporting performance (Gabbett, 2016). In general, $A C W R s \geq 1.5$ (i.e., greater acute than chronic training load) are associated with a higher injury risk than ACWRs in the range from 0.8 to 1.3 . While most studies showed the association of Acute:Chronic markers with risk of injuries, its application in predictions of injuries is questioned (Fanchini et al., 2018; McCall et al., 2018). Rather, it can identify workloads that athletes should use to make a decision when risk of injury is increased or decreased (Hulin and Gabbett, 2019). Therefore, the ACWR should not be viewed in isolation.

Contrary to these studies, the suggested association between training loads and back pain and/or injury is based on case studies, expert opinions, and unpublished data, whereas some other findings are controversial. Analysis of the literature identified several limitations in relation to workloads and back problems in athletes. First, the studies that use the same methods are limited. Second, injury identification is often based only on players' self-assessment. Third, the exposure hours are calculated as average values for training and playing, and in most cases are based on data reported by individuals. Additionally, prevalence of back pain and/or injury in some sports can be affected by sample size. Finally, numbers of injury definitions and time occurrence categories make a comparison among studies more difficult. Further studies using a wider methodological approach are necessary to deepen the knowledge and understanding the association between the training load, especially ACWR and back pain and/or injury in athletes of individual and team sports.

Because questionnaires are mainly used for identification of back pain, more objective methods evaluating spine curvature and flexibility (Muyor et al., 2017), strength, power and endurance of core muscles (Zemková et al., 2016a, 2017, 2019a,b; Zemková and Jeleň, 2019) and hamstrings (Zemková et al., 2015), as well as postural and core stability after perturbations (Zemková et al., 2016b) should be applied. In addition to sportspecific testing methods, basic physical fitness tests can be used (Zemková and Hamar, 2018). Athletes undergoing the high-level of training demonstrated similar deconditioning of the lumbar extensor muscles and used similar strategies to maintain spinal stability after unexpected perturbations to ensure the spine from pain and damage as compared to non-athletes with low back pain (Catalá et al., 2018). Assessment of training interventions for strengthening the core muscles is also necessary, not only in adult athletes with a predisposition to back pain and/or injury but also in adolescents (Zemková and Hamar, 2018). There is increasing evidence that an experience of back pain in 14year-old individuals may be associated with pain in adulthood (Coenen et al., 2017). Repetitive flexion and extension loadings of young Functional Spinal Units causes MRI and histological changes in the growth zones and endplates that are most likely first signs of fatigue and an explanation for the spine injuries in adolescent athletes (Thoreson et al., 2017a). In spite of the fact that back pain and/or injury is one of the most prevalent diagnosis in athletes, the mechanism of back problems is poorly understood so far (Ball et al., 2019). Research studies conducted on athletes with back pain highlight physiological and biomechanical mechanisms as influential, whereas psychological 
factors are often neglected. Heidari et al. (2017) emphasizes the importance of mainly stress-related factors in prevention and rehabilitation of back pain. Therefore, more longitudinal studies are required in comparison with frequently conducted shortterm experiments in order to investigate long-term changes in strength of core muscles and neuromuscular control of spine stability in athletes.

Collectivelly, the association between the ACWR and injury in a variety of sports has been well established. However, our review highlights the paucity of research studies directly evaluating the association between the ACWR and back pain and/or injury across a larger range of sports. The inconsistency in the methodology of calculating weekly training loads and missing specification of injuries are main limiting factors that restricted comparison between findings. To reduce the variability between studies, researchers need to clearly define the ACWR methodology and specify back problems. The acute and chronic time periods used for assessment of the ACWR may depend on the specific nature of particular sports. Future investigations would benefit from more information on the fundamentals of competition and training in particular sports, physical fitness attributes of athletes, and other back pain and/or injury risk factors.

\section{CONCLUSION}

Although the relationship exists between the training load and the occurrence of back problems in athletes of individual and team sports, this is not specifically related to ACWR. In comparison with biomechanical, physiological or stress-related psychological factors, sport-specific patterns underlying high prevalence rates are not fully elucidated. The fatigue of trunk muscles induced by excessive loading of the spine is one of the sources of back problems in athletes. Practitioners can address this issue by load management via the use of the ACWR. However, they should be aware that the acute and chronic time

\section{REFERENCES}

Aasa, U., Svartholm, I., Andersson, F., and Berglund, L. (2017). Injuries among weightlifters and powerlifters: a systematic review. Br. J. Sports Med. 51, 211219. doi: 10.1136/bjsports-2016-096037

Aasheim, C., Stavenes, H., Andersson, S. H., Engbretsen, L., and Clarsen, B. (2018). Prevalence and burden of overuse injuries in elite junior handball. BMJ Open Sport Exerc. Med. 4:e000391. doi: 10.1136/bmjsem-2018000391

Adiele, D., and Morgan, G. P. (2018). Prevalence of musculoskeletal injuries in males and females practicing swimming from higher school of Zimbabwe. Am. J. Sports Sci. 6, 8-11. doi: 10.11648/j.ajss.20180601.12

Airaksinen, O., Brox, J. I., Cedraschi, C., Hildebrandt, J., Klaber-Moffett, J., Kovacs, F., et al. (2006). Chapter 4. European guidelines for the management of chronic nonspecific low back pain. Eur. Spine J. 15, S192-S300. doi: 10.1007/s00586006-1072-1

Almeida, G. P. L., de Souza, V. L., Sano, S. S., Saccol, M. F., and Cohen, M. (2012). Comparison of hip rotation range of motion in judo athletes with and without history of low back pain. Man. Ther. 17, 231-235. doi: 10.1016/j.math.2012. 01.004 window may vary according to specific structure of individual and team sports and their training and competition schedules. Though this may provide important feedback on athlete's workload, the questionable is the sensitivity and specificity of the ACWR to predict back problems. This is mainly due to the limited evidence on applications of this systems-model approach for identification of risk of back pain and back injuries. Future research should focus on investigation of ACWR validity in combination with multifaceted monitoring systems in prevention of back problems in athletes of both individual and team sports. Nonetheless, the ACWR can be used as an effective tool for monitoring the athlete's responses to training thereby enable practitioners to design workloads that decrease the occurrence of back problems. It seems that high chronic workloads and adequate muscle strength and endurance have a protective effect on increasing the occurrence of sport-related injuries.

\section{DATA AVAILABILITY STATEMENT}

The raw data supporting the conclusions of this article will be made available by the authors, without undue reservation, to any qualified researcher.

\section{AUTHOR CONTRIBUTIONS}

All authors listed have made a substantial, direct and intellectual contribution to the work, and approved it for publication.

\section{FUNDING}

This work was supported by the Cross-border Co-operation Programme INTERREG V-A SK-CZ/2018/06 (No. 304011P714) co-financed by the European Regional Development Fund, and the Slovak Research and Development Agency under the contract No. APVV-15-0704.

Alricsson, M., Björklund, G., Cronholm, M., Olsson, O., Viklund, P., and Svantesson, U. (2016). Spinal alignment, mobility of the hip and thoracic spine and prevalence of low back pain in young elite cross-country skiers. J. Exerc. Rehab. 12, 21-28. doi: 10.12965/jer.150255

Alzahrani, H., Shirley, D., Cheng, S. W. M., Mackey, M., and Stamatakis, E. (2019). Physical activity and chronic back conditions: a population-based pooled study of 60,134 adults. J. Sport Health Sci. 8, 386-393. doi: 10.1016/j.jshs.2019.01.003

Angoules, A. G., Dionyssiotis, Y., Angoules, G. A., Balakatounis, K. C., Panou, A., and Papathanasiou, J. (2018). An epidemiological study of non-specific low back pain in non-professional female greek classic ballet dancers. Folia Med. 60, 248-253. doi: 10.1515/folmed-2017-0087

Anza, R., Denis, M., and Silva, M. F. (2013). Analysis of physical fitness, anthropometry and prevalence of musculoskeletal symptoms in the youth volleyball category. Rev. Bras. Med. Esporte 19, 62-65.

Armstrong, R., Hall, B. J., Doyle, J., and Waters, E. (2011). Cochrane update. 'Scoping the scope' of a cochrane review. J. Public Health (Oxf) 33, 147-150. doi: 10.1093/pubmed/fdr015

Augustsson, S. R., Augustsson, J., Thomee, R., and Svantesson, U. (2006). Injuries and preventive actions in elite Swedish volleyball. Scand. J. Med. Sci. Sports 16, 433-440. doi: 10.1111/j.1600-0838.2005.00517.x 
Bacon, C. S., and Mauger, A. R. (2017). Prediction of overuse injuries in professional u18-u21 footballers using metrics of training distance and intensity. J. Strength Cond. Res. 31, 3067-3076. doi: 10.1519/JSC. 0000000000001744

Bahr, R., and Reeser, J. C. (2003). Injuries among world-class professional beach volleyball players: the federation internationale de volleyball beach volleyball injury study. Am. J. Sports Med. 31, 119-125. doi: 10.1177/ 03635465030310010401

Ball, J. R., Harris, C. B., Lee, J., and Vives, M. J. (2019). Lumbar spine injuries in sports: review of the literature and current treatment recommendations. Sports Med. Open 5:26. doi: 10.1186/s40798-019-0199-7

Banister, E. W., Calvert, T. W., Savage, M. V., and Bach, T. (1975). A systems model of training for athletic performance. Austr. J. Sports Med. Exerc. Sci. 7, 57-61.

Baranto, A., Hellström, M., Nyman, R., Lundin, O., and Swärd, L. (2006). Back pain and degenerative abnormalities in the spine of young elite divers. Knee Surg. Sport Tr. A 14, 907-914. doi: 10.1007/s00167-005-0032-3

Belavý, D. L., Quittner, M. J., Ridgers, N., Ling, Y., Connell, D., and Rantalainen, T. (2017). Running exercise strengthens the intervertebral disc. Sci. Rep. 7:45975. doi: $10.1038 /$ srep 45975

Bere, T., Kruczynski, J., Veintimilla, N., Hamu, Y., and Bahr, R. (2015). Injury risk is low among world-class volleyball players: 4-year data from the FIVB injury surveillance system. Br. J. Sports Med. 49, 1132-1137. doi: 10.1136/bjsports2015-094959

Bovens, A. M. P., Janssen, G. M. E., Vermeer, H. G. W., Hoeberigs, J. H., Janssen, M. P. E., and Verstappen, F. T. J. (1989). Occurrence of running injuries in adults following a supervised training program. Int. J. Sport Med. 10, 186-190. doi: 10.1055/s-2007-1024970

Bowen, L., Gross, A. S., Gimpel, M., and Li, F. X. (2017). Accumulated workloads and the acute:chronic workload ratio relate to injury risk in elite youth football players. Br. J. Sports Med. 51, 452-459. doi: 10.1136/bjsports-2015-095820

Bromley, S. J., Drew, M. K., Talpey, S., McIntosh, A. S., and Finch, C. F. (2018). A systematic review of prospective epidemiological research into injury and illness in Olympic combat sport. Br. J. Sports Med. 52, 8-16. doi: 10.1136/bjsports2016-097313

Caine, D. J., and Nassar, L. (2005). Gymnastics injuries. Med. Sport Sci. 48, 18-58. doi: $10.1159 / 000084282$

Catalá, M. M., Schroll, A., Laube, G., and Arampatzis, A. (2018). Muscle strength and neuromuscular control in low-back pain: elite athletes versus general population. Front. Neurosci. 12:436. doi: 10.3389/fnins.2018.00436

Coenen, P., Smith, A., Paananen, M., O'Sullivan, P., Beales, D., and Straker, L. (2017). Trajectories of low back pain from adolescence to young adulthood. Arthritis Care Res. 69, 403-412. doi: 10.1002/acr.22949

Cole, M. H., and Grimshaw, P. N. (2016). The biomechanics of the modern golf swing: implications for lower back injuries. Sports Med. 46, 339-351. doi: 10. 1007/s40279-015-0429-1

Cross, M. J., Williams, S., Trewartha, G., Kemp, S. P., and Stokes, K. A. (2016). The influence of in-season training loads on injury risk in professional rugby union. Int. J. Sports Physiol. Perform. 11, 350-355. doi: 10.1123/ijspp.2015-0187

Daniels, J. M., Arguelles, C., Gleason, C., and Dixon, W. H. (2020). Back injuries. Prim. Care 47, 147-164. doi: 10.1016/j.pop.2019.10.008

Dennis, R. J., Finch, C. F., and Farhart, P. J. (2005). Is bowling workload a risk factor for injury to Australian junior cricket fast bowlers? Br. J. Sports Med. 39 , 843-846. doi: 10.1136/bjsm.2005.018515

Ekstrand, J. (1982). Football Injuries and Their Prevention. Medical Dissertation No 130. Linköping: Linköping University.

Fanchini, M., Rampinini, E., Riggio, M., Coutts, A., Pecci, C., and McCall, A. (2018). Despite association, the acute:chronic work load ratio does not predict non-contact injury in elite footballers. Sci. Med. Football 2, 108-114. doi: 10. 1080/24733938.2018.1429014

Farahbakhsh, F., Akbari-Fakhrabadi, M., Shariat, A., Cleland, J. A., Farahbakhsh, F., Seif-Bargh, T., et al. (2018). Neck pain and low back pain in relation to functional disability in different sport activities. J. Exerc. Rehabil. 14, 509-515. doi: $10.12965 /$ jer. 1836220.110

Fett, D., Trompeter, K., and Platen, P. (2017). Back pain in elite sports: a crosssectional study on 1114 athletes. PLoS One 12:e0180130. doi: 10.1371/journal. pone. 0180130

Fett, D., Trompeter, K., and Platen, P. (2019). Prevalence of back pain in a group of elite athletes exposed to repetitive overhead activity. PLoS One 14:e0210429. doi: 10.1371 /journal.pone. 0210429
Foster, C., Snyder, A., and Welsh, R. (1999). "Monitoring of training, warm up, and performance in athletes," in Overload, Performance Incompetence and Regeneration in Sport, eds M. Lehmann, C. Foster, U. Gastmann, H. Keizer, and J. M. Steinacker (New York, NY: Kluwer Academic Plenum), 43-51. doi: 10.1007/978-0-585-34048-7_4

Gabbett, T. J. (2004a). Influence of training and match intensity on injuries in rugby league. J. Sports Sci. 22, 409-417. doi: 10.1080/02640410310001641638

Gabbett, T. J. (2004b). Reductions in pre-season training loads reduce training injury rates in rugby league players. Br. J. Sports Med. 38, 743-749. doi: 10.1136/ bjsm.2003.008391

Gabbett, T. J. (2016). The training injury prevention paradox: should athletes be training smarter and harder? Br. J. Sports Med. 50, 273-280. doi: 10.1136/ bjsports-2015-095788

Gabbett, T. J., and Domrow, N. (2005). Risk factors for injury in subelite rugby league players. Am. J. Sports Med. 33, 428-434. doi: 10.1177/0363546504268407

Gabbett, T. J., and Domrow, N. (2007). Relationships between training load, injury, and fitness in sub-elite collision sport athletes. J. Sports Sci. 25, 1507-1519. doi: 10.1080/02640410701215066

Gabbett, T. J., and Ullah, S. (2012). Relationship between running loads and softtissue injury in elite team sport athletes. J. Strength Cond. Res. 26, 953-960. doi: 10.1519/JSC.0b013e3182302023

Gabbett, T. J., Ullah, S., and Finch, C. F. (2012). Identifying risk factors for contact injury in professional rugby league players-application of a frailty model for recurrent injury. J. Sci. Med. Sport 15, 496-504. doi: 10.1016/j.jsams.2012. 03.017

Gastin, P. B., Meyer, D., Huntsman, E., and Cook, J. (2015). Increase in injury risk with low body mass and aerobic-running fitness in elite Australian football. Int. J. Sports Physiol. Perform. 10, 458-463. doi: 10.1123/ijspp.2014-0257

Goldstein, J. D., Berger, P. E., Windler, G. E., and Jackson, D. W. (1991). Spine injuries in gymnasts and swimmers: an epidemiologic investigation. Am. J. Sports Med. 19, 463-468. doi: 10.1177/036354659101900507

Goosey-Tolfrey, V. (2010). Supporting the paralympic athlete: focus on wheeled sports. Disabil. Rehabil. 32, 2237-2243. doi: 10.3109/09638288.2010.491577

Graw, B. P., and Wiesel, S. W. (2008). Low back pain in the aging athlete. Sports Med. Arthrosc. Rev. 16, 39-46. doi: 10.1097/JSA.0b013e318163be67

Griffin, A., Kenny, I. C., Comyns, T. M., and Lyons, M. (2020). The association between the acute:chronic workload ratio and injury and its application in team sports: a systematic review. Sports Med. 50, 561-580. doi: 10.1007/s40279-01901218-2

Haag, T. B., Mayer, H. M., Schneider, A. S., Rumpf, M. C., Handel, M., and Schneider, C. (2016). Risk assessment of back pain in youth soccer players. Res. Sports Med. 24, 395-406. doi: 10.1080/15438627.2016.1222275

Haydt, R., Pheasant, S., and Lawrence, K. (2012). The incidence of low back pain in NCAA Division III female field hockey players. Int. J. Sports Phys. Ther. 7, 296-305.

Heidari, J., Hasenbring, M., Kleinert, J., and Kellmann, M. (2017). Stress-related psychological factors for back pain among athletes: important topic with scarce evidence. Eur. J. Sport Sci. 17, 351-359. doi: 10.1080/17461391.2016.1252429

Hoskins, W., Pollard, H., Daff, C., Odell, A., Garbutt, P., Mchardy, A., et al. (2009). Low back pain status in elite and semi-elite Australian football codes: a cross-sectional survey of football (soccer), Australian rules, rugby league, rugby union and non-athletic controls. BMC Musculoskelet. Disord. 10:38. doi: 10.1186/1471-2474-12-158

Hulin, B. T., and Gabbett, T. J. (2019). Indeed association does not equal prediction: the never-ending search for the perfect acute:chronic workload ratio. Br. J. Sports Med. 53, 144-145. doi: 10.1136/bjsports-2018-099448

Hulin, B. T., Gabbett, T. J., Blanch, P., Chapman, P., Bailey, D., and Orchard, J. W. (2014). Spikes in acute workload are associated with increased injury risk in elite cricket fast bowlers. Br. J. Sports Med. 48, 708-712. doi: 10.1136/bjsports-2013092524

Hulin, B. T., Gabbett, T. J., Caputi, P., Lawson, D. W., and Sampson, J. A. (2016a). Low chronic workload and the acute:chronic workload ratio are more predictive of injury than between-match recovery time: a two-season prospective cohort study in elite rugby league players. Br. J. Sports Med. 50, 1008-1012. doi: 10. 1136/bjsports-2015-095364

Hulin, B. T., Gabbett, T. J., Lawson, D. W., Caputi, P., and Sampson, J. A. (2016b). The acute:chronic workload ratio predicts injury: high chronic workload may decrease injury risk in elite rugby league players. Br. J. Sports Med. 50, 231-236. doi: 10.1136/bjsports-2015-094817 
Hunter, J. S. (1986). The exponentially weighted moving average. J. Qual. Technol. $18,203-210$

Jacobson, I., and Tegner, Y. (2007). Injuries among Swedish female elite football players: a prospective population study. Scand. J. Med. Sci. Sports 17, 84-91. doi: $10.1111 / j .1600-0838.2006 .00524 . x$

Jobson, S. A., Passfield, L., Atkinson, G., Barton, G., and Scarf, P. (2009). The analysis and utilization of cycling training data. Sports Med. 39, 833-844. doi: 10.2165/11317840-000000000-00000

Joeng, H. S., Na, Y. M., Lee, S. Y., and Cho, Y. J. (2018). Injuries among Korean female professional golfers: a prospective study. J. Sports Sci. Med. 17, 492-500.

Jonasson, P., Halldin, K., Karlsson, J., Thoreson, O., Hvannberg, J., Swärd, L., et al. (2011). Prevalence of joint-related pain in the extremities and spine in five groups of top athletes. Knee Surg. Sport Tr. A. 19, 1540-1546. doi: 10.1007/ s00167-011-1539-4

Junior, L. C. H., Costa, L. O. P., and Lopes, A. D. (2013). Previous injuries and some training characteristics predict running-related injuries in recreational runners: a prospective cohort study. J. Physiother. 59, 263-269. doi: 10.1016/ S1836-9553(13)70203-0

Kaneoka, K., Shimizu, K., Hangai, M., Okuwaki, T., Mamizuka, N., Sakane, M., et al. (2007). Lumbar intervertebral disk degeneration in elite competitive swimmers: a case control study. Am. J. Sports Med. 8, 1341-1345. doi: 10.1177/ 0363546507300259

Koes, B. W., van Tulder, M. W., and Peul, W. C. (2007). Diagnosis and treatment of sciatica. BMJ 334, 1313-1317. doi: 10.1136/bmj.39223.428495.BE

Koyama, K., Nakazato, K., Min, S. K., Gushiken, K., Hatakeda, Y., Seo, K., et al. (2013). Radiological abnormalities and low back pain in gymnasts. Int. J. Sports Med. 34, 218-222. doi: 10.1055/s-0032-1316366

Kraft, C. N., Pennekamp, P. H., Becker, U., Young, M., Diedrich, O., Lüring, C., et al. (2009). Magnetic resonance imaging findings of the lumbar spine in elite horseback riders: correlations with back pain, body mass index, trunk/leglength coefficient, and riding discipline. Am. J. Sports Med. 11, 2205-2213. doi: $10.1177 / 0363546509336927$

Külling, F. A., Florianz, H., Reepschläger, B., Gasser, J., Jost, B., and Lajtai, G. (2014). High prevalence of disc degeneration and spondylolysis in the lumbar spine of professional beach volleyball players. Orthop. J. Sports Med. 2:2325967114528862. doi: $10.1177 / 2325967114528862$

Lawrence, J. P., Greene, H. S., and Grauer, J. N. (2006). Back pain in athletes. J. Am. Acad. Orthop. 14, 726-735. doi: 10.5435/00124635-200612000-00004

Leppänen, M., Pasanen, K., Kujala, U. M., and Parkkari, J. (2015). Overuse injuries in youth basketball and floorball. Open Access J. Sports Med. 6, 173-179. doi: 10.2147/OAJSM.S82305

Lyman, S., Fleisig, G. S., Waterbor, J. W., Funkhouser, E. M., Pulley, L., Andrews, J. R., et al. (2001). Longitudinal study of elbow and shoulder pain in youth baseball pitchers. Med. Sci. Sports Exerc. 33, 1803-1810. doi: 10.1097/00005768200111000-00002

Macedo, L. G., Saragiotto, B. T., Yamato, T. P., Costa, L. O. P., Menezes Costa, L. C., Ostelo, R. W. J. G., et al. (2016). Motor control exercise for acute nonspecific low back pain. Cochrane Database Syst. Rev. 2:CD012085. doi: 10.1002/ 14651858.CD012085

Macera, C. A., Pate, R. R., Powell, K. E., Jackson, K. L., Kendrick, J. S., and Craven, T. E. (1989). Predicting lower-extremity injuries among habitual runners. Arch. Intern. Med. 149, 2565-2568. doi: 10.1001/archinte.149.11. 2565

Malone, S., Roe, M., Doran, D. A., Gabbett, T. J., and Collins, K. D. (2017a). Aerobic fitness and playing experience protect against spikes in workload: the role of the acute:chronic workload ratio on injury risk in elite Gaelic football. Int. J. Sports Physiol. Perform. 12, 393-401. doi: 10.1123/ijspp.2016-0090

Malone, S., Roe, M., Doran, D., Gabbett, T. J., and Collins, K. (2017b). High chronic training loads and exposure to bouts of maximal velocity running reduce injury risk in Gaelic football. J. Sci. Med. Sport 20, 250-254. doi: 10.1016/j.jsams.2016. 08.005

Maselli, F., Ciuro, A., Mastrosimone, R., Cannone, M., Nicoli, P., Signori, A., et al. (2015). Low back pain among Italian rowers: a cross-sectional survey. J. Back Muskuloskelet. 28, 365-376. doi: 10.3233/BMR-140529

McCall, A., Dupont, G., and Ekstrand, J. (2018). Internal workload and noncontact injury: a one-season study of five teams from the UEFA elite club injury study. Br. J. Sports Med. 52, 1517-1522. doi: 10.1136/bjsports-2017-0 98473
McHardy, A., Pollard, H., and Luo, K. (2007). One-year follow-up study on golf injuries in Australian amateur golfers. Am. J. Sports Med. 35, 1354-1360. doi: $10.1177 / 0363546507300188$

Menaspa, P. (2017). Are rolling averages a good way to assess training load for injury prevention? Br. J. Sports Med. 51, 618-619. doi: 10.1136/bjsports-2016096131

Moradi, V., Memari, A. H., ShayestehFar, M., and Kordi, R. (2015). Low back pain in athletes is associated with general and sport specific risk factors: a comprehensive review of longitudinal studies. Rehabil. Res. Pract. 2015:850184. doi: $10.1155 / 2015 / 850184$

Murray, E., Birley, E., Twycross-Lewis, R., and Morrissey, D. (2009). The relationship between hip rotation range of movement and low back pain prevalence in amateur golfers: an observational study. Phys. Ther. Sport 10, 131-135. doi: 10.1016/.pptsp.2009.08.002

Murray, N. B., Gabbett, T. J., Townshend, A. D., Hulin, B. T., and McLellan, C. P. (2017). Individual and combined effects of acute and chronic running loads on injury risk in elite Australian footballers. Scand. J. Med. Sci. Sports 27, 990-998. doi: $10.1111 /$ sms. 12719

Muyor, J. M., López-Miñarro, P. A., and Alacid, F. (2011). Spinal posture of thoracic and lumbar spine and pelvic tilt in highly trained cyclists. J. Sports Sci. Med. 10, 355-361.

Muyor, J. M., Zemková, E., and Chren, M. (2017). Effects of Latin style professional dance on the spinal posture and pelvic tilt. J. Back Musculoskelet. Rehabil. 30, 791-800. doi: 10.3233/BMR-150448

Newlands, C., Reid, D., and Parmar, P. (2015). The prevalence, incidence and severity of low back pain among international-level rowers. Br. J. Sports Med. 49, 951-956. doi: 10.1136/bjsports-2014-093889

Nosco, D. L., Kim Carpenter, D. C., and Zhang, J. (1999). Determination of risk factors for low back pain in female adolescent volleyball players. Int. J.Volleyball Res. 7, 33-42.

Orchard, J. W., James, T., Portus, M., Kountouris, A., and Dennis, R. (2009). Fast bowlers in cricket demonstrate up to 3- to 4-week delay between high workloads and increased risk of injury. Am. J. Sports Med. 37, 1186-1192. doi: $10.1177 / 0363546509332430$

Pasanen, K., Parkkari, J., Kannus, P., Rossi, L., Palvanen, M., Natri, A., et al. (2008). Injury risk in female floorball: a prospective one-season follow-up. Scand. J. Med. Sci. Sports 18, 49-54. doi: 10.1111/j.1600-0838.2007.00640.x

Peebles, R., and Jonas, C. E. (2017). Sacroiliac joint dysfunction in the athlete: diagnosis and management. Curr. Sports Med. Rep. 16, 336-342. doi: 10.1249/ JSR.0000000000000410

Piazza, M., Di Cagno, A., Cupisti, A., Panicucci, E., and Santoro, G. (2009). Prevalence of low back pain in former rhythmic gymnasts. J. Sports Med. Phys. Fitness 49, 297-300

Piotrowska, S. E., Majchrzycki, M., Rogala, P., and Mazurek-Sitarz, M. (2017). Lower extremity and spine pain in cyclists. Ann. Agric. Environ. Med. 24, 654-658. doi: 10.5604/12321966.1233552

Plais, N., Salzmann, S. N., Shue, J., Sanchez, C. D., Urraza, F. J., and Girardi, F. P. (2019). Spine injuries in soccer. Curr. Sports Med. Rep. 18, 367-373. doi: 10.1249/JSR.0000000000000638

Poór, O., and Zemková, E. (2018). The effect of training in the preparatory and competitive periods on trunk rotational power in canoeists, ice-hockey players and tennis players. Sports (Basel) 6, E113. doi: 10.3390/sports6040113

Quarrie, K. L., Alsop, J. C., Waller, A. E., Bird, Y. N., Marshall, S. W., and Chalmers, D. J. (2001). The New Zealand rugby injury and performance project. VI. A prospective cohort study of risk factors for injury in rugby union football. Br. J. Sports Med. 35, 157-166. doi: 10.1136/bjsm.35.3.157

Reis, F. J., Dias, M. D., Newlands, F., Meziat-Filho, N., and Macedo, A. R. (2015). Chronic low back pain and disability in Brazilian jiu-jitsu athletes. Phys. Ther. Sport 16, 340-343. doi: 10.1016/j.ptsp.2015.02.005

Rostami, M., Ansari, M., Noormohammadpour, P., Mansournia, M. A., and Kordi, R. (2015). Ultrasound assessment of trunk muscles and back flexibility, strength and endurance in off-road cyclists with and without low back pain. J. Back Musculoskelet. Rehabil. 28, 635-644. doi: 10.3233/BMR- 140559

Roussel, N., De Kooning, M., Schutt, A., Mottram, S., Truijen, S., Nijs, J., et al. (2013). Motor control and low back pain in dancers. Int. J. Sports Med. 34, 138-143. doi: 10.1055/s-0032-1321722

Ruckstuhl, L., and Clénin, G. (2019). Back pain and core strength in elite cycling. Schweiz Z. Med. Traumatol. 67, 44-48. 
Samuelsson, K., Larsson, H., Thyberg, M., and Gerdle, B. (2001). Wheelchair seating intervention. Results from a client-centred approach. Disabil. Rehabil. 23, 677-682. doi: 10.1080/09638280110049900

Saraceni, N., Kemp-Smith, K., O'Sullivan, P., and Campbell, A. (2017). The relationship between lead hip rotation and low back pain in golfers - a pilot investigation. Int. J. Golf Sci. 6, 130-141. doi: 10.1123/ijgs.2017-0014

Saragiotto, B. T., Maher, C. G., Yamato, T. P., Costa, L. O. P., Menezes Costa, L. C., Ostelo, R. W. J. G., et al. (2016). Motor control exercise for chronic non-specific low-back pain. Cochrane Database Syst. Rev. 1, CD012004. doi: 10.1002/14651858.CD012004

Schultz, S. J., and Gordon, S. J. (2010). Recreational cyclists: the relationship between low back pain and training characteristics. Int. J. Exerc. Sci. 3, 79-85.

Sekine, C., Hirayama, K., Yanagisawa, O., Okubo, Y., Hangai, M., Imai, A., et al. (2014). Lumbar intervertebral disc degeneration in collegiate rowers. J. Phys. Fitness Sports Med. 3, 525-530. doi: 10.7600/jpfsm.3.525

Siewe, J., Rudat, J., Röllinghoff, M., Schlegel, U. J., Eysel, P., and Michael, J. P. (2011). Injuries and overuse syndromes in powerlifting. Int. J. Sports Med. 32, 703-711. doi: 10.1055/s-0031-1277207

Smith, B. E., Littlewood, C., and May, S. (2014). An update of stabilisation exercises for low back pain: a systematic review with meta-analysis. BMC Musculoskelet. Disord. 15:416. doi: 10.1186/1471-2474-15-416

Snellman, K., Parkkari, J., Kannus, P., Leppälä, J., Vuori, I., and Järvinen, M. (2001). Sports injuries in floorball: a prospective one-year follow-up study. Int. J. Sports Med. 22, 531-536. doi: 10.1055/s-2001-17609

Soligard, T., Schwellnus, M., Alonso, J. M., Bahr, R., Clarsen, B., Dijkstra, H. P., et al. (2016). How much is too much? (Part 1) international olympic committee consensus statement on load in sport and risk of injury. Br. J. Sports Med. 50, 1030-1041. doi: 10.1136/bjsports-2016-096581

Taha, T., and Thomas, S. G. (2003). Systems modelling of the relationship between training and performance. Sports Med. 33, 1061-1073. doi: 10.2165/00007256200333140-00003

Tertti, M., Paajanen, H., Kujala, U. M., Alanen, A., Salmi, T. T., and Kormano, M. (1990). Disc degeneration in young gymnasts: a magnetic resonance imaging study. Am. J. Sports Med. 18, 206-208. doi: 10.1177/036354659001800216

Tesarz, J., Gerhardt, A., Schommer, K., Treede, R. D., and Eich, W. (2013). Alterations in endogenous pain modulation in endurance athletes: an experimental study using quantitative sensory testing and the cold-pressor task. Pain 154, 1022-1029. doi: 10.1016/j.pain.2013.03.014

Tesarz, J., Schuster, A. K., Hartmann, M., Gerhardt, A., and Eich, W. (2012). Pain perception in athletes compared to normally active controls: a systematic review with meta-analysis. Pain 153, 1253-1262. doi: 10.1016/j.pain.2012.03.005

Thoreson, O., Ekström, L., Hansson, H. A., Todd, C., Witwit, W., Aminoff, A. S., et al. (2017a). The effect of repetitive flexion and extension fatigue loading on the young porcine lumbar spine, a feasibility study of MRI and histological analyses. J. Exp. Orthop. 4:16. doi: 10.1186/s40634-017-0091-7

Thoreson, O., Kovac, P., Swärd, A., Agnvall, C., Todd, C., and Baranto, A. (2017b). Back pain and MRI changes in the thoraco-lumbar spine of young elite Mogul skiers. Scand. J. Med. Sci. Sports 27, 983-989. doi: 10.1111/sms.12710

Trainor, T. J., and Wiesel, S. W. (2002). Epidemiology of back pain in the athlete. Clin. Sports Med. 21, 93-103. doi: 10.1016/s0278-5919(03)00059-0

Trompeter, K., Fett, D., and Platen, P. (2017). Prevalence of back pain in sports: a systematic review of the literature. Sports Med. 47, 1183-1207. doi: 10.1007/ s40279-016-0645-3

Trompeter, K., Fett, D., and Platen, P. (2019). Back pain in rowers: a crosssectional study on prevalence, pain characteristics and risk factors. Sportverletz Sportschaden. 33, 51-59. doi: 10.1055/a-0648-8387

Tunås, P., Nilstad, A., and Myklebust, G. (2015). Low back pain in female elite football and handball players compared with an active control group. Knee Surg. Sports Traumatol. Arthrosc. 23, 2540-2547. doi: 10.1007/s00167-014-3069-3

van Hilst, J., Hilgersom, N. F., Kuilman, M. C., Kuijer, P. P. F. M., and Frings-Dresen, M. H. W. (2015). Low back pain in young elite field hockey players, football players and speed skaters: prevalence and risk factors. J. Back Musculoskelet. 28, 67-73. doi: 10.3233/BMR-140491

van Middelkoop, M., Kolkman, J., van Ochten, J., Bierma-Zeinstra, S. M. A., and Koes, B. W. (2007). Course and predicting factors of lower-extremity injuries after running a marathon. Clin. J. Sport Med. 17, 25-30. doi: 10.1097/JSM. $0 \mathrm{~b} 013 \mathrm{e} 3180305 \mathrm{e} 4 \mathrm{~d}$ van Middelkoop, M., Kolkman, J., van Ochten, J., Bierma-Zeinstra, S. M. A., and Koes, B. W. (2008). Risk factors for lower extremity injuries among male marathon runners. Scand. J. Med. Sci. Sports 18, 691-697. doi: 10.1111/j.16000838.2007.00768.x

Villavicencio, A. T., Burneikienë, S., Hernández, T. D., and Thramann, J. (2006). Back and neck pain in triathletes. Neurosurg. Focus 21, 1-7. doi: 10.3171/foc. 2006.21.4.8

Wilber, C. A., Holland, G. J., Madison, R. E., and Loy, S. F. (1995). An epidemiological analysis of overuse injuries among recreational cyclists. Int. J. Sport Med. 16, 201-206. doi: 10.1055/s-2007-972992

Williams, S., West, S., Cross, M. J., and Stokes, K. A. (2017). Better way to determine the acute:chronic workload ratio? Br. J. Sports Med. 51, 209-210. doi: 10.1136/bjsports-2016-096589

Windt, J., Gabbett, T. J., Ferris, D., and Khan, K. M. (2017). Training load-injury paradox: is greater preseason participation associated with lower in-season injury risk in elite rugby league players? Br. J. Sports Med. 51, 645-650. doi: 10.1136/bjsports-2016-095973

Wirth, K., Hartmann, H., Mickel, C., Szilvas, E., Keiner, M., and Sander, A. (2017). Core stability in athletes: a critical analysis of current guidelines. Sports Med. 47, 401-414. doi: 10.1007/s40279-016-0597-7

Witwit, W. A., Kovac, P., Sward, A., Agnvall, C., Todd, C., Thoreson, O., et al. (2018). Disc degeneration on MRI is more prevalent in young elite skiers compared to controls. Knee Surg. Sport Tr. A. 26, 325-332. doi: 10.1007/s00167017-4545-3

Zemková, E., Cepková, A., Uvaček, M., and Hamar, D. (2016a). A new method to assess the power performance during a lifting task in young adults. Measurement 91, 460-467. doi: 10.1016/j.measurement.2016.05.077

Zemková, E., Cepková, A., Uvaček, M., and Šooš, L' (2017). A novel method for assessing muscle power during the standing cable wood chop exercise. J. Strength Cond. Res. 31, 2246-2254. doi: 10.1519/JSC.0000000000001692

Zemková, E., and Hamar, D. (2018). Sport-specific assessment of the effectiveness of neuromuscular training in young athletes. Front. Physiol. 9:264. doi: 10.3389/ fphys.2018.00264

Zemková, E., and Jeleň, M. (2019). Differentiation of the strength of back muscle contraction under fatigue: Does force feedback play a role? J. Sport Rehabil. doi: 10.1123/jsr.2018-0496 [Epub ahead of print].

Zemková, E., Jeleň, M., and Zapletalová, L. (2018a). Trunk rotational velocity in young and older adults: a role of trunk angular displacement. Res. Phys. Educ. Sport Health 7, 103-107.

Zemková, E., Miklovič, P., Dunajčík, A., and Hamar, D. (2015). Power as a parameter in the functional assessment of knee flexions and knee extensions on weight stack machines. Measurement 61, 141-149.

Zemková, E., Muyor, J. M., and Jeleň, M. (2018b). Association of trunk rotational velocity with spine mobility and curvatures in para table tennis players. Int. J. Sports Med. 39, 1055-1062. doi: 10.1055/a-0752-4224

Zemková, E., Poór, O., and Jeleň, M. (2019a). Between-side differences in trunk rotational power in athletes trained in asymmetric sports. J. Back Musculoskelet. Rehabil. 32, 529-537. doi: 10.3233/BMR-181131

Zemková, E., Poór, O., and Pecho, J. (2019b). Peak rate of force development and isometric maximum strength of back muscles are associated with power performance during load-lifting tasks. Am. J. Men's Health 13, 1-8. doi: 10.1177/ 1557988319828622

Zemková, E., Štefániková, G., and Muyor, J. M. (2016b). Load release balance test under unstable conditions effectively discriminates between physically active and sedentary young adults. Hum. Mov. Sci. 48, 142-152. doi: 10.1016/j.humov. 2016.05.002

Conflict of Interest: The authors declare that the research was conducted in the absence of any commercial or financial relationships that could be construed as a potential conflict of interest.

Copyright (C) 2020 Zemková, Kováčiková and Zapletalová. This is an open-access article distributed under the terms of the Creative Commons Attribution License (CC BY). The use, distribution or reproduction in other forums is permitted, provided the original author(s) and the copyright owner(s) are credited and that the original publication in this journal is cited, in accordance with accepted academic practice. No use, distribution or reproduction is permitted which does not comply with these terms. 